\title{
Identification of target associations for polypharmacology from analysis of crystallographic ligands of the Protein Data Bank
}

\author{
Luca Pinzi $i^{\dagger}, \underline{\text { Giulio Rastelli }}{ }^{\dagger}, *$ \\ $\dagger$ Department of Life Sciences, University of Modena and Reggio Emilia, Via Giuseppe Campi \\ 103, 41125 Modena, Italy.
}

\footnotetext{
* Correspondence to: Prof. Giulio Rastelli, Department of Life Sciences, University of Modena and Reggio Emilia, Via Giuseppe Campi 103, 41125 Modena, Italy. Tel +39 0352058564 , Emailgiulio.rastelli@unimore.it
} 
Figure S1: ROCS-based alignments of the I0G and 4NA crystallographic ligands. The I0G and 4NA ligands are represented as green and cyan sticks, respectively. A visual inspection of the obtained alignments revealed that the (3as,4r,9br)-4-(4-hydroxyphenyl)-1,2,3,3a,4,9bhexahydrocyclopenta[c]chromen-8-ol and 1-chloro-6-(4-hydroxyphenyl)-2-naphthol chemical moieties of I0G and 4NA well overlapped each other, providing similar steric hindrance and shape properties despite being structurally unrelated. Moreover, the two distal hydroxyl groups in the ligands, which engage H-bond interactions with key residues in their respective crystal complexes, perfectly overlapped each other in the obtained alignments. ${ }^{1,2}$

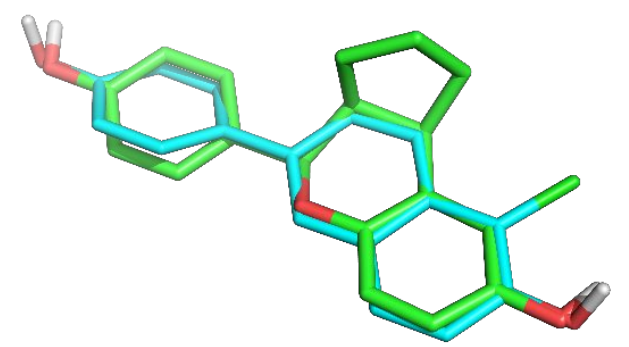


Figure S2: Frequency distributions of the identified percentages of sequence similarity and identity. In particular, panel a shows the distribution of the percentages of sequence similarity and identity by considering all the identified target associations reported in Table S2. Panel $\boldsymbol{b}$ shows the distribution of the percentages of sequence similarity and identity of target associations with already reported common therapeutic indications (reported in Table 4). Distributions of the percentage of similarity and identity are represented in blue and light orange, respectively.

a)

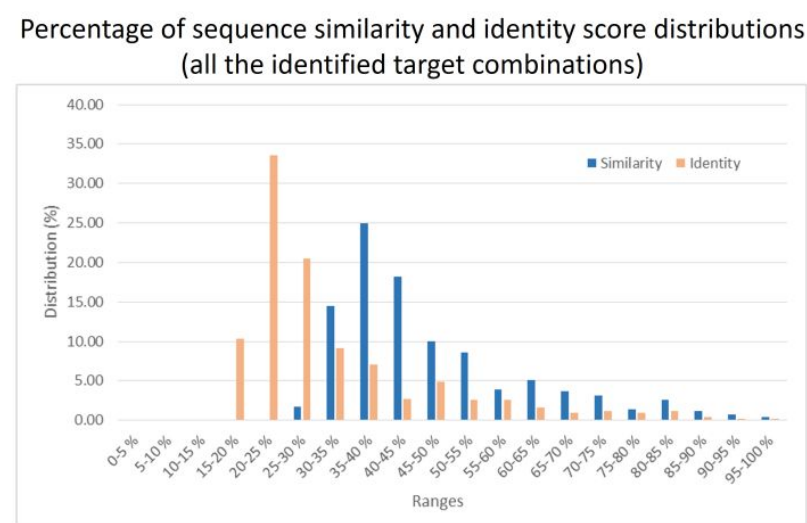

b)

Percentage of sequence similarity and identity score distributions (target combinations reported in Table 4)

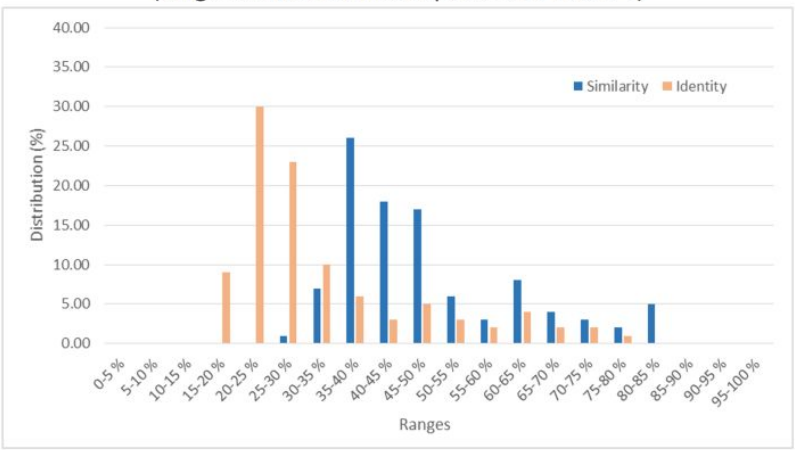


Figure S3: Predicted docking poses of LY2, 8DQ, BXZ, 272, RRC and P01 crystallographic ligands. In particular, panel $a$ reports the predicted binding modes of the 8DQ and LY2 compounds into the 1YI3 and 4CFL crystal structures, respectively. Panel $b$ reports the predicted binding modes of BXZ and 272 into the 1U3Q and 3BM9 crystal structures, respectively. Panel $c$ reports the predicted binding modes of the P01 and RRC into the $1 \mathrm{UNL}$ and $1 Y O M$ crystal structures, respectively. Residue numbering refers to that of the crystal structures employed in the docking calculations. The crystallographic conformations and predicted poses of the ligands are colored in deepteal and orange, respectively.

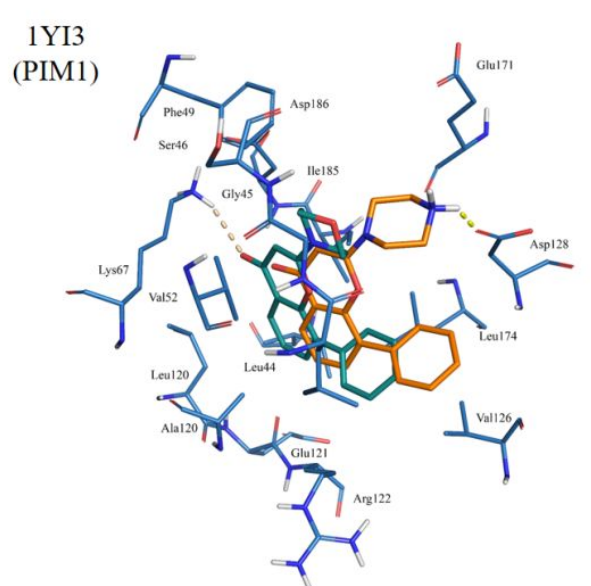

a)
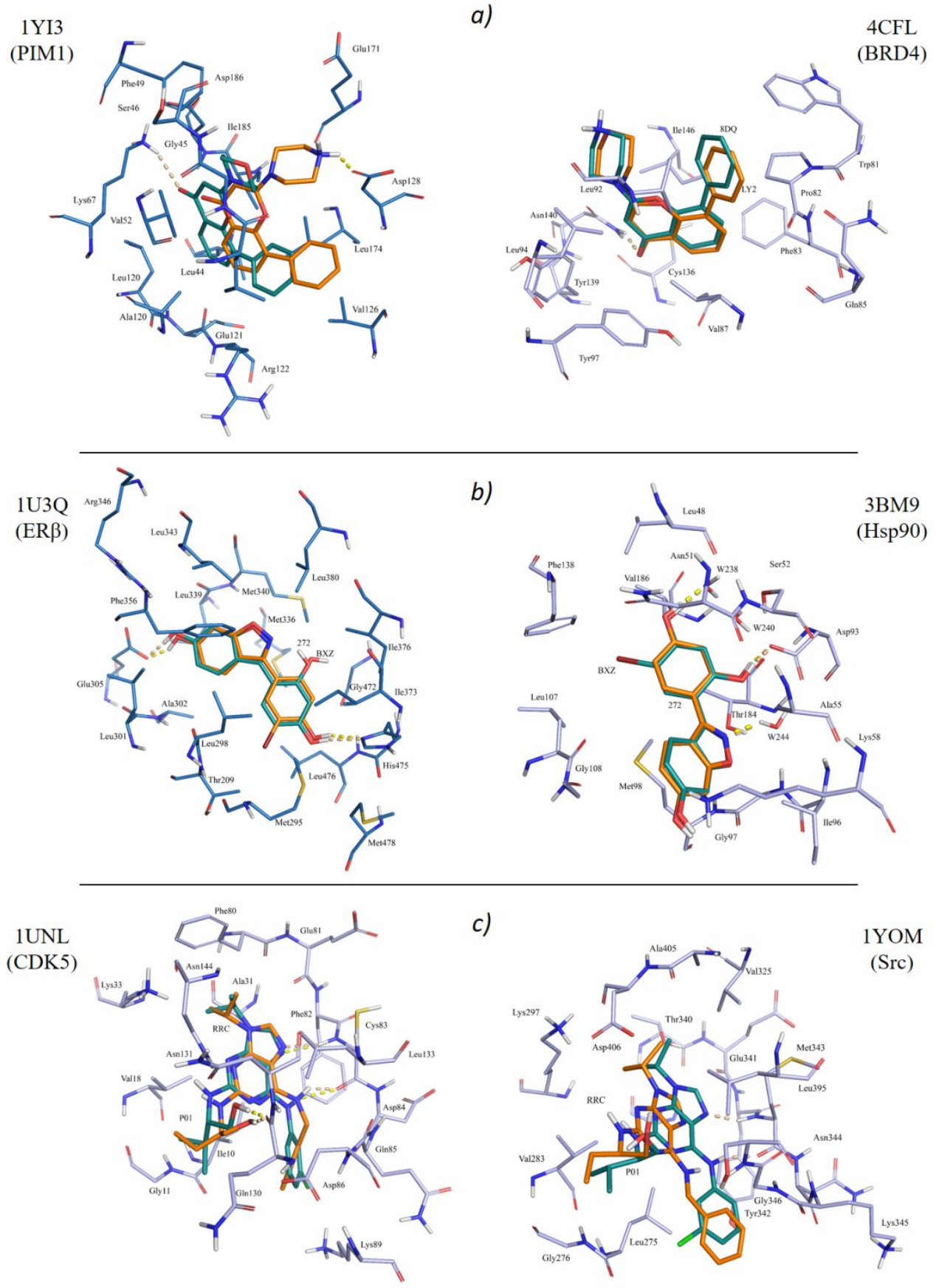

c)

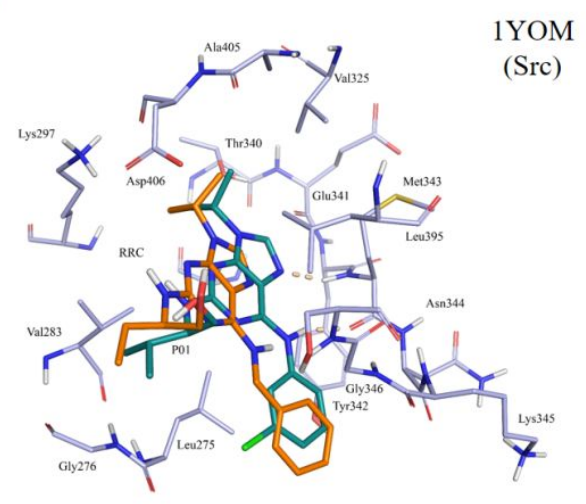


Figure S4: Superimposition of the 2BRH (CHK1) and 4JQ7 (EGFR) binding sites. The binding site residues of $\mathbf{2 B R H}$ and $\mathbf{4 J Q 7}$ are represented in green and cyan sticks, respectively. Binding site residues that differ across the two proteins are labeled in red.

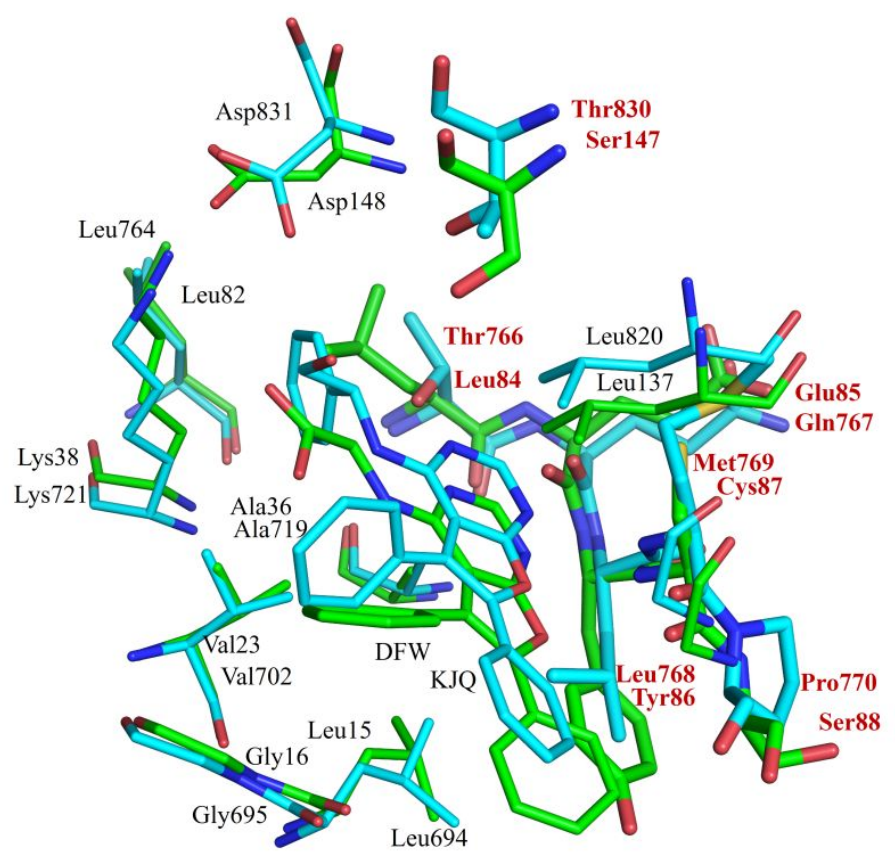


Figure S5: Dose-response curve of the DFW and Staurosporine (control) compounds on wild type EGFR.
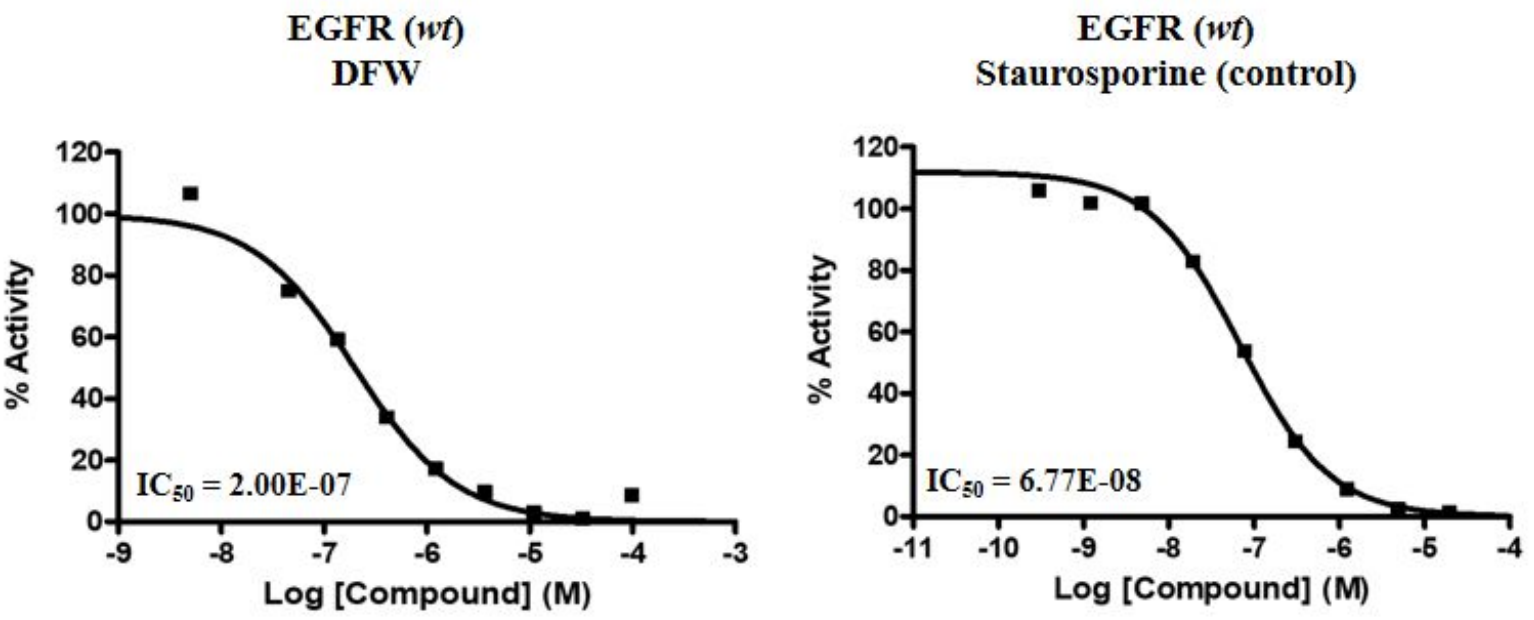
Table S1: Representative examples of pair of ligands identified as similar solely by one of the ROCS, MACCS $f p$ and ECFP4 $f p$ ligand-based methods. The first three examples (example IDs 1 to 3) refer to pair of ligands identified as similar only by MACCS fingerprints (MACCS $f p$ Tanimoto score higher than 0.8). Example IDs 4 to 6 refer to pair of ligands identified as similar only by ECFP4 fingerprints (ECFP4fp Tanimoto score $\geq 0.3$ ). Examples 7 to 9 refer to pair of similar ligands identified only by ROCS fingerprints (TanimotoCombo $\geq 1.5$ ). Scores higher than the employed thresholds of similarity are highlighted in bold.

\begin{tabular}{|c|c|c|c|c|c|c|c|c|c|c|c|}
\hline $\begin{array}{c}\text { Example } \\
\text { ID }\end{array}$ & $\begin{array}{c}P D B \\
\text { complex } \\
1\end{array}$ & $\begin{array}{c}\text { LIG } \\
\text { complex } \\
1\end{array}$ & $\begin{array}{c}\text { 2D Structure } \\
\text { Complex } 1\end{array}$ & $\begin{array}{c}P D B \\
\text { complex } \\
2\end{array}$ & $\begin{array}{c}\text { LIG } \\
\text { complex } \\
2\end{array}$ & $\begin{array}{c}2 D \text { Structure } \\
\text { Complex } 2\end{array}$ & $\begin{array}{c}\text { ROCS } \\
\text { Tanimoto } \\
\text { Combo }\end{array}$ & $\begin{array}{c}\text { MACCSfp } \\
\text { score }\end{array}$ & $\begin{array}{c}\text { ECFP4fp } \\
\text { score }\end{array}$ & $\begin{array}{c}\text { Uniprot } \\
\text { Name } \\
\text { complex } \\
1\end{array}$ & $\begin{array}{c}\text { Uniprot } \\
\text { Name } \\
\text { complex } \\
2\end{array}$ \\
\hline 1 & $3 \mathrm{~W} 16$ & P9J & & $2 \mathrm{~W} 1 \mathrm{~F}$ & LOF & & 1.004 & 0.851 & 0.237 & $\begin{array}{l}\text { AURKA } \\
\text { HUMAN }\end{array}$ & $\begin{array}{l}\text { AURKA } \\
\text { HUMAN }\end{array}$ \\
\hline 2 & $3 \mathrm{P} 8 \mathrm{X}$ & ZYD & & 3AUR & CA9 & & 1.248 & 0.917 & 0.238 & $\begin{array}{c}\text { VDR } \\
\text { HUMAN }\end{array}$ & $\begin{array}{c}\text { VDR } \\
\text { HUMAN }\end{array}$ \\
\hline 3 & $1 \times 7 \mathrm{~J}$ & GEN & & 4DEU & NAR & & 1.152 & 0.862 & 0.235 & $\begin{array}{c}\text { ER } \beta \\
\text { HUMAN }\end{array}$ & $\begin{array}{c}\text { TTHY } \\
\text { HUMAN }\end{array}$ \\
\hline 4 & $3 \mathrm{SBH}$ & E65 & & 4MTY & SBW & & 1.243 & 0.614 & 0.955 & $\begin{array}{c}\text { CAH2 } \\
\text { HUMAN }\end{array}$ & $\begin{array}{c}\text { CAH2 } \\
\text { HUMAN }\end{array}$ \\
\hline
\end{tabular}


Z87

$6 \quad 3 R N I$

217

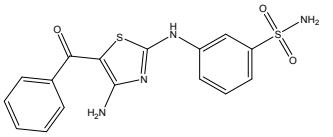

7

2I0G

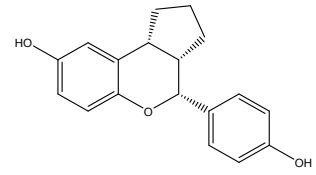

105A

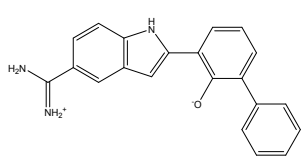

1GJ8

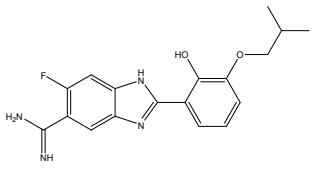

4BU3

3AMA

$1 Y Y 4$

4NA

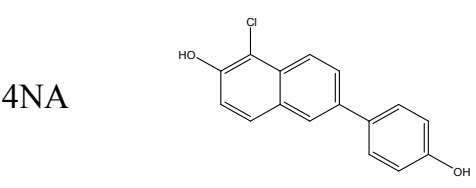

133

SKE

9 3KR8 XAV
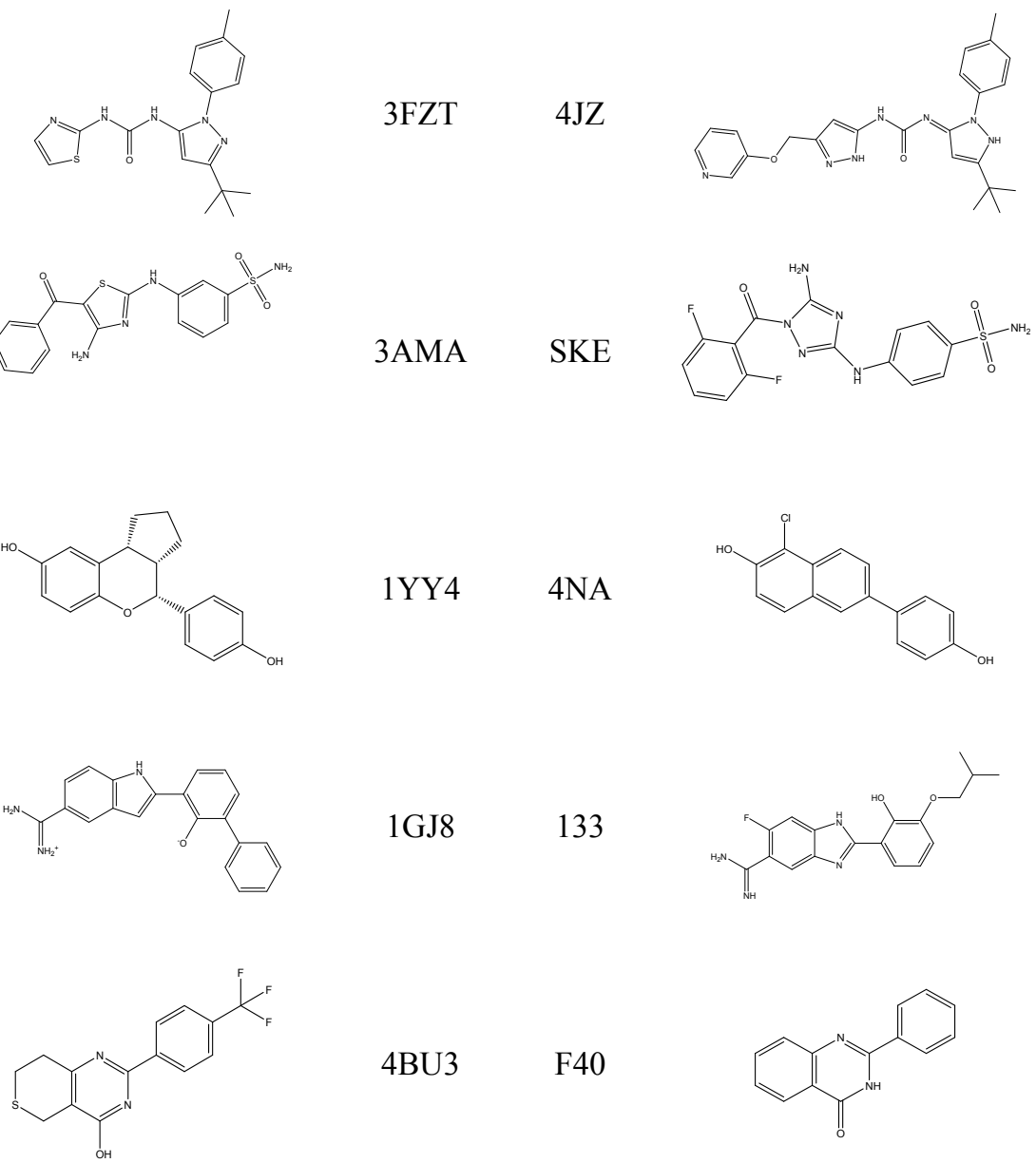

1.506
F40

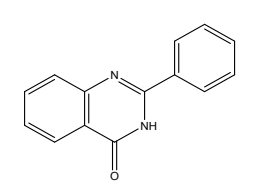
XAV

0.916

0.661

1.238

0.571

0.787

0.840

CDK2 KAPCA HUMAN HUMAN
1.661
MK14 HUMAN HUMAN

$\operatorname{ER} \beta$ $\operatorname{ER} \beta$ HUMAN HUMAN

1.537 0.641 0.282

UROK UROK HUMAN HUMAN

TNKS2 TNKS2 HUMAN HUMAN 
Table S2: Target associations identified by the ligand-based comparisons with unreported common therapeutic indications. Target combinations for which at least one pair provided unsuccessful redocking are shown in red. Protein names are based on UNIPROT IDs.

\begin{tabular}{|c|c|c|c|c|c|c|c|}
\hline $\begin{array}{l}\text { UNIPROT NAME } \\
\text { Protein } 1\end{array}$ & $\begin{array}{l}\text { UNIPROTNAME } \\
\text { Protein } 2\end{array}$ & $\begin{array}{c}\text { Number of } \\
\text { reported duals } \\
\text { (actives) }^{a}\end{array}$ & $\begin{array}{c}\text { Number of reported } \\
\text { duals (similar to the } \\
\text { actives) }\end{array}$ & $\begin{array}{l}\text { known - } \\
\text { known }\end{array}$ & $\begin{array}{l}\text { known - } \\
\text { unknown }\end{array}$ & $\begin{array}{c}\text { unknown - } \\
\text { unknown }\end{array}$ & $\begin{array}{l}\text { Ligands with dual } \\
\text { activity (actives) }\end{array}$ \\
\hline XIAP_HUMAN & BIRC2_HUMAN & $548(416)$ & $1056(765)$ & 4 & 2 & 0 & $7(3)$ \\
\hline WEE1_HUMAN & PIM1_HUMAN & $107(4)$ & 0 & 0 & 0 & 1 & 0 \\
\hline VTDB_HUMAN & VDR_HUMAN & $2(1)$ & $4(2)$ & 0 & 1 & 0 & $1(1)$ \\
\hline VRK1_HUMAN & AVR2A_HUMAN & $6(0)$ & 0 & 0 & 0 & 1 & 0 \\
\hline VGFR2_HUMAN & CDK8_HUMAN & $114(10)$ & $4(4)$ & 1 & 0 & 0 & $2(0)$ \\
\hline UPP1_HUMAN & UPP2_HUMAN & 0 & 0 & 1 & 0 & 0 & 0 \\
\hline ULK1_HUMAN & KCC2D_HUMAN & $88(10)$ & $2(0)$ & 0 & 0 & 2 & 0 \\
\hline ULK1_HUMAN & STK26_HUMAN & $91(14)$ & $2(0)$ & 0 & 0 & 1 & 0 \\
\hline TYK2_HUMAN & DAPK3_HUMAN & $138(20)$ & $2(0)$ & 1 & 0 & 0 & $2(0)$ \\
\hline TYK2_HUMAN & JAK1_HUMAN & $1222(766)$ & $37(34)$ & 3 & 0 & 0 & $6(6)$ \\
\hline TYK2_HUMAN & PKN1_HUMAN & $128(17)$ & $2(2)$ & 1 & 0 & 0 & $2(2)$ \\
\hline TTK_HUMAN & ITK_HUMAN & $374(18)$ & $6(0)$ & 0 & 1 & 1 & 0 \\
\hline TTHY_HUMAN & AK1BA_HUMAN & $8(3)$ & $14(6)$ & 3 & 1 & 0 & $5(4)$ \\
\hline TTHY_HUMAN & ALBU_HUMAN & $6(1)$ & $16(0)$ & 2 & 2 & 0 & $4(0)$ \\
\hline TTHY_HUMAN & ESR1_HUMAN & $7(2)$ & $6(4)$ & 2 & 0 & 0 & $2(1)$ \\
\hline TTHY_HUMAN & FABPH_HUMAN & 0 & 0 & 1 & 0 & 0 & 0 \\
\hline TTHY_HUMAN & S10A7_HUMAN & $1(0)$ & $2(0)$ & 1 & 0 & 0 & $2(1)$ \\
\hline TTHY_HUMAN & ESR2_HUMAN & $3(2)$ & $4(4)$ & 1 & 0 & 0 & $2(1)$ \\
\hline TTHY_HUMAN & FTO_HUMAN & 0 & 0 & 0 & 2 & 0 & 0 \\
\hline TTHY_HUMAN & ALDR_HUMAN & $14(3)$ & 0 & 0 & 1 & 0 & 0 \\
\hline TTHY_HUMAN & PTGR2_HUMAN & 0 & 0 & 1 & 0 & 0 & 0 \\
\hline TNKS2_HUMAN & PAR15_HUMAN & $6(0)$ & 0 & 1 & 0 & 0 & 0 \\
\hline TNKS2_HUMAN & AURKA_HUMAN & $9(2)$ & $14(8)$ & 0 & 0 & 2 & 0 \\
\hline TNKS2 HUMAN & BRD2 HUMAN & 0 & 0 & 0 & 0 & 2 & 0 \\
\hline
\end{tabular}




\begin{tabular}{|c|c|c|c|c|c|c|c|}
\hline $\begin{array}{c}\text { UNIPROT NAME } \\
\text { Protein } 1\end{array}$ & $\begin{array}{c}\text { UNIPROTNAME } \\
\text { Protein } 2\end{array}$ & $\begin{array}{l}\text { Number of } \\
\text { reported duals } \\
\text { (actives) }^{a}\end{array}$ & $\begin{array}{c}\text { Number of reported } \\
\text { duals (similar to the } \\
\text { actives) }\end{array}$ & $\begin{array}{l}\text { known - } \\
\text { known }\end{array}$ & $\begin{array}{l}\text { known - } \\
\text { unknown }\end{array}$ & $\begin{array}{l}\text { unknown - } \\
\text { unknown }\end{array}$ & $\begin{array}{l}\text { Ligands with dual } \\
\text { activity (actives) }\end{array}$ \\
\hline TNKS1_HUMAN & PAR15_HUMAN & $6(0)$ & 0 & 1 & 0 & 0 & 0 \\
\hline TNKS1_HUMAN & TNKS2_HUMAN & $389(333)$ & $110(96)$ & 3 & 2 & 0 & $8(8)$ \\
\hline TNIK_HUMAN & PIM1_HUMAN & $199(8)$ & $2(0)$ & 0 & 0 & 1 & 0 \\
\hline TNIK_HUMAN & WEE1_HUMAN & $99(16)$ & 0 & 1 & 0 & 0 & 0 \\
\hline THBG_HUMAN & TTHY_HUMAN & $5(1)$ & $2(0)$ & 0 & 1 & 0 & $1(1)$ \\
\hline THBG_HUMAN & AK1C2_HUMAN & $1(1)$ & $2(2)$ & 1 & 0 & 0 & $2(2)$ \\
\hline THB_HUMAN & TTHY_HUMAN & $26(3)$ & $4(4)$ & 1 & 0 & 0 & $2(0)$ \\
\hline TAB1_HUMAN & FAK1_HUMAN & $9(2)$ & $1(1)$ & 0 & 0 & 1 & 0 \\
\hline SYFM_HUMAN & PH4H_HUMAN & 0 & 0 & 1 & 0 & 0 & 0 \\
\hline STK26_HUMAN & CDKL2_HUMAN & $87(12)$ & 0 & 1 & 0 & 0 & 0 \\
\hline STK26_HUMAN & VRK1_HUMAN & $79(0)$ & 0 & 0 & 0 & 1 & 0 \\
\hline STK25_HUMAN & KCC1G_HUMAN & $160(9)$ & $8(4)$ & 1 & 0 & 0 & $2(2)$ \\
\hline STK25_HUMAN & PAK6_HUMAN & $189(9)$ & $8(2)$ & 1 & 0 & 0 & $2(2)$ \\
\hline STK24_HUMAN & CHK1_HUMAN & $278(12)$ & 0 & 0 & 1 & 0 & 0 \\
\hline STK10_HUMAN & ERBB3_HUMAN & $106(18)$ & $5(5)$ & 1 & 1 & 0 & $3(3)$ \\
\hline ST17B_HUMAN & VRK1_HUMAN & $7(0)$ & 0 & 0 & 0 & 1 & 0 \\
\hline SRPK2_HUMAN & KS6A1_HUMAN & $275(12)$ & $7(0)$ & 0 & 1 & 0 & $1(1)$ \\
\hline SRC_HUMAN & STK10_HUMAN & $568(36)$ & $5(5)$ & 1 & 1 & 0 & $3(3)$ \\
\hline SRC_HUMAN & SRPK2_HUMAN & $281(12)$ & $5(0)$ & 0 & 1 & 0 & $1(0)$ \\
\hline SRC_HUMAN & KS6A1_HUMAN & $807(26)$ & 10 & 1 & 0 & 0 & $2(2)$ \\
\hline SLK_HUMAN & CLK3_HUMAN & $227(13)$ & $2(0)$ & 1 & 0 & 0 & $2(2)$ \\
\hline SLK_HUMAN & CDKL2_HUMAN & $89(21)$ & 0 & 1 & 0 & 0 & 0 \\
\hline SLK_HUMAN & STK26_HUMAN & $205(23)$ & $2(0)$ & 1 & 0 & 0 & $2(2)$ \\
\hline SIR1_HUMAN & SIR3_HUMAN & $416(94)$ & $23(0)$ & 0 & 1 & 0 & $1(0)$ \\
\hline S10A7_HUMAN & CDK2_HUMAN & $1(0)$ & $2(0)$ & 1 & 0 & 0 & $2(2)$ \\
\hline S10A4_HUMAN & TNNC1_HUMAN & 0 & 0 & 1 & 1 & 0 & 0 \\
\hline RXRA_HUMAN & FTO_HUMAN & 0 & 0 & 1 & 0 & 0 & 0 \\
\hline RXRA_HUMAN & PPARG_HUMAN & $13(1)$ & 0 & 1 & 0 & 0 & 0 \\
\hline RORG_HUMAN & NPC1_HUMAN & 0 & 0 & 1 & 1 & 0 & 0 \\
\hline RORG_HUMAN & CP11A HUMAN & 0 & 0 & 0 & 0 & 1 & 0 \\
\hline
\end{tabular}




\begin{tabular}{|c|c|c|c|c|c|c|c|}
\hline $\begin{array}{c}\text { UNIPROT NAME } \\
\text { Protein } 1\end{array}$ & $\begin{array}{c}\text { UNIPROTNAME } \\
\text { Protein } 2\end{array}$ & $\begin{array}{l}\text { Number of } \\
\text { reported duals } \\
\text { (actives) }^{a}\end{array}$ & $\begin{array}{c}\text { Number of reported } \\
\text { duals (similar to the } \\
\text { actives) }^{a}\end{array}$ & $\begin{array}{l}\text { known - } \\
\text { known }\end{array}$ & $\begin{array}{l}\text { known - } \\
\text { unknown }\end{array}$ & $\begin{array}{l}\text { unknown - } \\
\text { unknown }\end{array}$ & $\begin{array}{l}\text { Ligands with dual } \\
\text { activity (actives) }\end{array}$ \\
\hline RORA_HUMAN & RORG_HUMAN & $109(37)$ & $4(4)$ & 0 & 0 & 1 & 0 \\
\hline RIPK2_HUMAN & D3DSX2_HUMAN & 0 & 0 & 1 & 0 & 0 & 0 \\
\hline RIPK2_HUMAN & FGFR4_HUMAN & $270(13)$ & $4(0)$ & 1 & 0 & 0 & 0 \\
\hline RIPK2_HUMAN & DDR1_HUMAN & $109(28)$ & $4(0)$ & 1 & 0 & 0 & 0 \\
\hline RET_HUMAN & LCK_HUMAN & $731(84)$ & $41(14)$ & 0 & 1 & 0 & $1(1)$ \\
\hline RET_HUMAN & HCK_HUMAN & $624(40)$ & $28(6)$ & 1 & 0 & 0 & 0 \\
\hline RET_HUMAN & CBR1_HUMAN & 0 & 0 & 0 & 0 & 1 & 0 \\
\hline RASH_HUMAN & PPIF_HUMAN & 0 & 0 & 0 & 0 & 1 & 0 \\
\hline RARB_HUMAN & RARA_HUMAN & 374 (149) & $67(12)$ & 0 & 1 & 0 & $1(1)$ \\
\hline RABP2_HUMAN & RARB_HUMAN & $2(1)$ & $1(0)$ & 0 & 1 & 0 & $1(1)$ \\
\hline QPCTL_HUMAN & QPCT_HUMAN & $14(13)$ & $2(2)$ & 1 & 0 & 0 & $2(2)$ \\
\hline PYGL_HUMAN & RIFK_HUMAN & 0 & 0 & 1 & 0 & 0 & 0 \\
\hline PTGR2_HUMAN & ALBU_HUMAN & 0 & 0 & 2 & 1 & 0 & 0 \\
\hline PTGR2_HUMAN & AK1C2_HUMAN & 0 & 0 & 1 & 0 & 0 & 0 \\
\hline PTGR1_HUMAN & ESR1_HUMAN & 0 & 0 & 1 & 2 & 0 & 0 \\
\hline PPIF_HUMAN & PPIA_HUMAN & $5(4)$ & 0 & 1 & 0 & 0 & 0 \\
\hline PPARG_HUMAN & TTHY_HUMAN & $3(0)$ & $2(0)$ & 1 & 1 & 0 & $1(1)$ \\
\hline PPARG_HUMAN & AK1C2_HUMAN & $1(0)$ & $2(0)$ & 1 & 0 & 0 & $2(2)$ \\
\hline PPARG_HUMAN & PTGR2_HUMAN & 0 & 0 & 1 & 0 & 0 & 0 \\
\hline PKN1_HUMAN & JAK2_HUMAN & $133(18)$ & $2(2)$ & 1 & 0 & 0 & $2(2)$ \\
\hline PK3C3_HUMAN & PK3CA_HUMAN & $78(24)$ & $7(3)$ & 0 & 1 & 0 & 0 \\
\hline PIM1_HUMAN & CLK3_HUMAN & $528(8)$ & 0 & 1 & 0 & 0 & 0 \\
\hline PIM1_HUMAN & DYR1A_HUMAN & $498(16)$ & $2(2)$ & 1 & 0 & 0 & $2(1)$ \\
\hline PIM1_HUMAN & PIM2_HUMAN & 2947 (2084) & $636(622)$ & 1 & 0 & 0 & $2(2)$ \\
\hline PH4H_HUMAN & TPH1_HUMAN & 0 & 0 & 1 & 2 & 0 & 0 \\
\hline PH4H_HUMAN & AL1L1_HUMAN & 0 & 0 & 0 & 0 & 1 & 0 \\
\hline PH4H_HUMAN & NOS3_HUMAN & 0 & 0 & 1 & 1 & 0 & 0 \\
\hline PH4H_HUMAN & NOS1_HUMAN & 0 & 0 & 1 & 1 & 0 & 0 \\
\hline PH4H_HUMAN & NOS2_HUMAN & 0 & 0 & 3 & 1 & 0 & 0 \\
\hline PDPK1_HUMAN & F16P1_HUMAN & $3(0)$ & 0 & 0 & 0 & 2 & 0 \\
\hline
\end{tabular}




\begin{tabular}{|c|c|c|c|c|c|c|c|}
\hline $\begin{array}{c}\text { UNIPROT NAME } \\
\text { Protein } 1\end{array}$ & $\begin{array}{c}\text { UNIPROTNAME } \\
\text { Protein } 2\end{array}$ & $\begin{array}{l}\text { Number of } \\
\text { reported duals } \\
\text { (actives) }^{a}\end{array}$ & $\begin{array}{c}\text { Number of reported } \\
\text { duals (similar to the } \\
\text { actives) }\end{array}$ & $\begin{array}{l}\text { known - } \\
\text { known }\end{array}$ & $\begin{array}{l}\text { known - } \\
\text { unknown }\end{array}$ & $\begin{array}{l}\text { unknown - } \\
\text { unknown }\end{array}$ & $\begin{array}{l}\text { Ligands with dual } \\
\text { activity (actives) }\end{array}$ \\
\hline PDK2_HUMAN & HS90A_HUMAN & $2(2)$ & $6(6)$ & 0 & 1 & 2 & $1(1)$ \\
\hline PDK1_HUMAN & CP2C9_HUMAN & $4(0)$ & 0 & 0 & 0 & 1 & 0 \\
\hline PDE9A_HUMAN & PDE4D_HUMAN & $93(3)$ & $4(0)$ & 1 & 0 & 0 & $2(2)$ \\
\hline PDE8A_HUMAN & PDE2A_HUMAN & $133(0)$ & $4(0)$ & 1 & 0 & 0 & $2(2)$ \\
\hline PDE8A_HUMAN & PDE5A_HUMAN & $142(3)$ & $4(0)$ & 1 & 0 & 0 & $2(2)$ \\
\hline PDE8A_HUMAN & PDE9A_HUMAN & $137(0)$ & $4(0)$ & 1 & 0 & 0 & $2(2)$ \\
\hline PDE7A_HUMAN & PDE8A_HUMAN & $112(3)$ & $8(6)$ & 1 & 0 & 0 & $2(2)$ \\
\hline PDE7A_HUMAN & PDE9A_HUMAN & $114(3)$ & $2(0)$ & 1 & 0 & 0 & $2(2)$ \\
\hline PDE7A_HUMAN & PDE4D_HUMAN & $226(39)$ & $18(0)$ & 1 & 0 & 0 & $2(2)$ \\
\hline PDE7A_HUMAN & PDE5A_HUMAN & $186(23)$ & $2(0)$ & 1 & 0 & 0 & $2(2)$ \\
\hline PDE5A_HUMAN & PDE9A_HUMAN & $170(21)$ & $6(0)$ & 1 & 0 & 0 & $2(2)$ \\
\hline PDE5A_HUMAN & PDE2A_HUMAN & $222(26)$ & $16(0)$ & 1 & 0 & 0 & $2(2)$ \\
\hline PDE5A_HUMAN & PDE3B_HUMAN & $354(40)$ & $120(20)$ & 1 & 0 & 0 & $2(1)$ \\
\hline PDE4D_HUMAN & PDE2A_HUMAN & $233(28)$ & $10(2)$ & 1 & 0 & 0 & $2(2)$ \\
\hline PDE4D_HUMAN & PDE8A_HUMAN & $84(1)$ & $4(0)$ & 1 & 0 & 0 & $2(1)$ \\
\hline PDE3B_HUMAN & PDE8A_HUMAN & $39(3)$ & $4(0)$ & 1 & 0 & 0 & $2(0)$ \\
\hline PDE3B_HUMAN & PDE9A_HUMAN & $41(2)$ & $4(0)$ & 1 & 0 & 0 & $2(1)$ \\
\hline PDE3B_HUMAN & PDE4D_HUMAN & $759(105)$ & $122(8)$ & 1 & 0 & 0 & $2(2)$ \\
\hline PDE3B_HUMAN & PDE2A_HUMAN & $240(20)$ & $14(0)$ & 1 & 0 & 0 & $2(2)$ \\
\hline PDE3B_HUMAN & PDE7A_HUMAN & $70(4)$ & $8(2)$ & 1 & 0 & 0 & $2(2)$ \\
\hline PDE2A_HUMAN & PDE9A_HUMAN & $149(3)$ & $6(0)$ & 1 & 0 & 0 & $2(2)$ \\
\hline PDE2A_HUMAN & PDE7A_HUMAN & $124(15)$ & $4(0)$ & 1 & 0 & 0 & $2(2)$ \\
\hline PDE10_HUMAN & PDE4D_HUMAN & $166(34)$ & $16(12)$ & 1 & 0 & 0 & $2(1)$ \\
\hline PARP2_HUMAN & PARP1_HUMAN & $390(190)$ & $230(194)$ & 3 & 0 & 0 & $6(6)$ \\
\hline PARP2_HUMAN & TNKS2_HUMAN & $171(78)$ & $34(30)$ & 0 & 1 & 0 & $1(0)$ \\
\hline PARP1_HUMAN & TNKS2_HUMAN & 237 (109) & $224(162)$ & 2 & 1 & 0 & $5(4)$ \\
\hline PAK6_HUMAN & PAK4_HUMAN & $423(15)$ & 0 & 1 & 0 & 0 & 0 \\
\hline PAK6_HUMAN & PAK1_HUMAN & $201(11)$ & 0 & 1 & 0 & 0 & 0 \\
\hline PAK5_HUMAN & PAK4_HUMAN & $425(20)$ & $12(2)$ & 1 & 0 & 0 & 0 \\
\hline PAK1_HUMAN & PAK4_HUMAN & $238(55)$ & $24(4)$ & 1 & 0 & 0 & $2(1)$ \\
\hline
\end{tabular}




\begin{tabular}{|c|c|c|c|c|c|c|c|}
\hline $\begin{array}{c}\text { UNIPROT NAME } \\
\text { Protein } 1\end{array}$ & $\begin{array}{c}\text { UNIPROTNAME } \\
\text { Protein } 2\end{array}$ & $\begin{array}{l}\text { Number of } \\
\text { reported duals } \\
\text { (actives) }^{a}\end{array}$ & $\begin{array}{c}\text { Number of reported } \\
\text { duals (similar to the } \\
\text { actives) }\end{array}$ & $\begin{array}{l}\text { known - } \\
\text { known }\end{array}$ & $\begin{array}{l}\text { known - } \\
\text { unknown }\end{array}$ & $\begin{array}{l}\text { unknown - } \\
\text { unknown }\end{array}$ & $\begin{array}{l}\text { Ligands with dual } \\
\text { activity (actives) }\end{array}$ \\
\hline OXDA_HUMAN & PH4H_HUMAN & 0 & 0 & 0 & 0 & 1 & 0 \\
\hline OXDA_HUMAN & SYFM_HUMAN & 0 & 0 & 0 & 0 & 1 & 0 \\
\hline NRK1_HUMAN & BST1_HUMAN & 0 & 0 & 1 & 0 & 0 & 0 \\
\hline NR1I2_HUMAN & NCOA1_HUMAN & 0 & 0 & 1 & 0 & 0 & 0 \\
\hline NR1H2_HUMAN & NR1H3_HUMAN & $731(524)$ & $70(56)$ & 2 & 0 & 0 & 0 \\
\hline NR1H2_HUMAN & CP11A_HUMAN & 0 & 0 & 0 & 0 & 1 & 0 \\
\hline NR1H2_HUMAN & RORA_HUMAN & $33(13)$ & 0 & 0 & 0 & 1 & 0 \\
\hline NR1H2_HUMAN & RORG_HUMAN & $35(13)$ & $16(10)$ & 1 & 0 & 1 & $2(2)$ \\
\hline NQO2_HUMAN & ABL2_HUMAN & $2(2)$ & $2(2)$ & 1 & 0 & 0 & $2(2)$ \\
\hline NQO2_HUMAN & DDR1_HUMAN & $2(2)$ & $2(2)$ & 1 & 0 & 0 & $2(2)$ \\
\hline NPC1_HUMAN & ADRB2_HUMAN & 0 & 0 & 1 & 1 & 0 & 0 \\
\hline NPC1_HUMAN & RORA_HUMAN & 0 & 0 & 1 & 1 & 0 & 0 \\
\hline NPC1_HUMAN & NR1H2_HUMAN & 0 & 0 & 0 & 0 & 2 & 0 \\
\hline NOS3_HUMAN & AL1L1_HUMAN & 0 & 0 & 0 & 0 & 1 & 0 \\
\hline NOS2_HUMAN & TPH1_HUMAN & 0 & 0 & 0 & 0 & 1 & 0 \\
\hline NOS2_HUMAN & NOS1_HUMAN & $981(355)$ & $9(3)$ & 1 & 1 & 0 & 0 \\
\hline NOS1_HUMAN & AL1L1_HUMAN & 0 & 0 & 0 & 0 & 1 & 0 \\
\hline NOS1_HUMAN & NOS3_HUMAN & $938(251)$ & $6(2)$ & 1 & 0 & 0 & 0 \\
\hline NMRL1_HUMAN & TTHY_HUMAN & 0 & 0 & 0 & 1 & 0 & 0 \\
\hline NMRL1_HUMAN & ANDR_HUMAN & 0 & 0 & 0 & 0 & 1 & 0 \\
\hline NMNA3_HUMAN & CD38_HUMAN & 0 & 0 & 1 & 0 & 0 & 0 \\
\hline NMNA3_HUMAN & NRK1_HUMAN & 0 & 0 & 1 & 0 & 0 & 0 \\
\hline NEP_HUMAN & LKHA4_HUMAN & $3(2)$ & $2(2)$ & 1 & 0 & 0 & $2(2)$ \\
\hline NEP_HUMAN & ECE1_HUMAN & $119(56)$ & $4(4)$ & 1 & 0 & 0 & $2(1)$ \\
\hline NEK2_HUMAN & KIT_HUMAN & $428(16)$ & $14(6)$ & 0 & 1 & 0 & $1(1)$ \\
\hline NEK1_HUMAN & CDK2_HUMAN & $359(3)$ & $12(0)$ & 1 & 1 & 0 & 0 \\
\hline NAMPT_HUMAN & CD38_HUMAN & 0 & 0 & 1 & 0 & 0 & 0 \\
\hline NAMPT_HUMAN & BST1_HUMAN & 0 & 0 & 1 & 0 & 0 & 0 \\
\hline NAMPT_HUMAN & NMNA3_HUMAN & 0 & 0 & 1 & 0 & 0 & 0 \\
\hline NAMPT_HUMAN & NRK1_HUMAN & 0 & 0 & 1 & 0 & 0 & 0 \\
\hline
\end{tabular}




\begin{tabular}{|c|c|c|c|c|c|c|c|}
\hline $\begin{array}{c}\text { UNIPROT NAME } \\
\text { Protein } 1\end{array}$ & $\begin{array}{c}\text { UNIPROTNAME } \\
\text { Protein } 2\end{array}$ & $\begin{array}{l}\text { Number of } \\
\text { reported duals } \\
\text { (actives) }^{a}\end{array}$ & $\begin{array}{c}\text { Number of reported } \\
\text { duals (similar to the } \\
\text { actives) }^{a}\end{array}$ & $\begin{array}{l}\text { known - } \\
\text { known }\end{array}$ & $\begin{array}{l}\text { known - } \\
\text { unknown }\end{array}$ & $\begin{array}{l}\text { unknown - } \\
\text { unknown }\end{array}$ & $\begin{array}{l}\text { Ligands with dual } \\
\text { activity (actives) }\end{array}$ \\
\hline MYLK4_HUMAN & NEK1_HUMAN & $94(6)$ & $4(0)$ & 0 & 0 & 1 & 0 \\
\hline MTOR_HUMAN & PK3C3_HUMAN & $61(13)$ & $3(1)$ & 0 & 1 & 0 & 0 \\
\hline MRCKB_HUMAN & ROCK1_HUMAN & $478(6)$ & $16(4)$ & 1 & 1 & 0 & $3(3)$ \\
\hline MRCKB_HUMAN & TYK2_HUMAN & $164(10)$ & $8(0)$ & 0 & 1 & 0 & $1(0)$ \\
\hline MRCKB_HUMAN & MK08_HUMAN & $289(12)$ & $2(0)$ & 0 & 1 & 0 & $1(0)$ \\
\hline MMP8_HUMAN & MMP1_HUMAN & $708(335)$ & $142(88)$ & 1 & 0 & 0 & $2(2)$ \\
\hline MMP8_HUMAN & MMP12_HUMAN & 267 (186) & $2(2)$ & 1 & 0 & 0 & 0 \\
\hline MMP8_HUMAN & MMP13_HUMAN & $679(508)$ & $154(73)$ & 1 & 0 & 0 & $2(2)$ \\
\hline MMP8_HUMAN & MMP16_HUMAN & $52(35)$ & $4(4)$ & 1 & 0 & 0 & 0 \\
\hline MMP3_HUMAN & MMP12_HUMAN & $312(105)$ & $324(280)$ & 2 & 6 & 0 & $10(4)$ \\
\hline MMP16_HUMAN & ATS5_HUMAN & $7(1)$ & $2(2)$ & 1 & 0 & 0 & 0 \\
\hline MMP12_HUMAN & MMP9_HUMAN & $394(123)$ & $105(92)$ & 1 & 1 & 0 & 0 \\
\hline MMP12_HUMAN & MMP16_HUMAN & $49(33)$ & $2(2)$ & 1 & 0 & 0 & 0 \\
\hline MMP1_HUMAN & MMP13_HUMAN & $1769(975)$ & $208(103)$ & 0 & 1 & 0 & $1(1)$ \\
\hline MK14_HUMAN & KC1D_HUMAN & $578(28)$ & $103(50)$ & 0 & 2 & 0 & $2(2)$ \\
\hline MK14_HUMAN & CDK8_HUMAN & $128(5)$ & $4(4)$ & 1 & 0 & 0 & $2(2)$ \\
\hline MK14_HUMAN & TTBK1_HUMAN & $8(0)$ & $1(0)$ & 0 & 0 & 1 & 0 \\
\hline MK11_HUMAN & ABL1_HUMAN & $584(15)$ & $2(2)$ & 1 & 0 & 0 & $2(1)$ \\
\hline MK11_HUMAN & MK14_HUMAN & $1476(464)$ & $24(24)$ & 1 & 1 & 0 & $3(3)$ \\
\hline MK10_HUMAN & TTK_HUMAN & $178(30)$ & $56(6)$ & 1 & 0 & 0 & $2(2)$ \\
\hline MK08_HUMAN & TYK2_HUMAN & $166(20)$ & $4(1)$ & 0 & 0 & 1 & 0 \\
\hline MK03_HUMAN & MK01_HUMAN & $1122(168)$ & $4(4)$ & 1 & 0 & 0 & $2(2)$ \\
\hline MIF_HUMAN & TTHY_HUMAN & 0 & 0 & 0 & 0 & 1 & 0 \\
\hline MIF_HUMAN & CAH2_HUMAN & $1(0)$ & 0 & 1 & 1 & 0 & 0 \\
\hline MIF_HUMAN & MK01_HUMAN & $1(1)$ & 0 & 1 & 0 & 0 & 0 \\
\hline MERTK_HUMAN & SRC_HUMAN & $448(32)$ & $4(0)$ & 1 & 0 & 0 & $2(0)$ \\
\hline MERTK_HUMAN & SRPK2_HUMAN & $273(13)$ & $3(0)$ & 0 & 1 & 0 & $1(0)$ \\
\hline MERTK_HUMAN & KC1G3_HUMAN & $416(7)$ & $4(0)$ & 1 & 0 & 0 & $2(0)$ \\
\hline MEN1_HUMAN & CP2D6_HUMAN & 0 & 0 & 0 & 0 & 1 & 0 \\
\hline MDM2_HUMAN & MDM4_HUMAN & $377(160)$ & $4(4)$ & 1 & 0 & 0 & $2(2)$ \\
\hline
\end{tabular}




\begin{tabular}{|c|c|c|c|c|c|c|c|}
\hline $\begin{array}{l}\text { UNIPROT NAME } \\
\text { Protein } 1\end{array}$ & $\begin{array}{c}\text { UNIPROTNAME } \\
\text { Protein } 2\end{array}$ & $\begin{array}{l}\text { Number of } \\
\text { reported duals } \\
\text { (actives) }^{a}\end{array}$ & $\begin{array}{c}\text { Number of reported } \\
\text { duals (similar to the } \\
\text { actives) }\end{array}$ & $\begin{array}{l}\text { known - } \\
\text { known }\end{array}$ & $\begin{array}{l}\text { known - } \\
\text { unknown }\end{array}$ & $\begin{array}{l}\text { unknown - } \\
\text { unknown }\end{array}$ & $\begin{array}{l}\text { Ligands with dual } \\
\text { activity (actives) }\end{array}$ \\
\hline MCR_HUMAN & AK1D1_HUMAN & 0 & 0 & 1 & 0 & 0 & 0 \\
\hline MAPK2_HUMAN & MAPK3_HUMAN & $539(4)$ & $30(18)$ & 3 & 0 & 0 & $4(4)$ \\
\hline LCK_HUMAN & KIT_HUMAN & $639(115)$ & $6(4)$ & 1 & 0 & 0 & $2(2)$ \\
\hline LCK_HUMAN & SRC_HUMAN & $1469(324)$ & $140(104)$ & 0 & 0 & 1 & 0 \\
\hline LCK_HUMAN & CBR1_HUMAN & $3(0)$ & 0 & 0 & 0 & 1 & 0 \\
\hline LCK_HUMAN & ABL2_HUMAN & $632(47)$ & $6(4)$ & 1 & 0 & 0 & $2(2)$ \\
\hline LCK_HUMAN & MK14_HUMAN & $1378(150)$ & $7(2)$ & 1 & 1 & 0 & $3(3)$ \\
\hline KS6A1_HUMAN & CDK2_HUMAN & $693(9)$ & $13(0)$ & 1 & 0 & 0 & $2(2)$ \\
\hline KS6A1_HUMAN & CDK5_HUMAN & $581(6)$ & $9(0)$ & 1 & 0 & 0 & $2(2)$ \\
\hline KS6A1_HUMAN & MERTK_HUMAN & $445(21)$ & $4(0)$ & 1 & 0 & 0 & $2(0)$ \\
\hline KS6A1_HUMAN & KC1G1_HUMAN & $579(11)$ & $5(0)$ & 1 & 0 & 0 & $2(1)$ \\
\hline KS6A1_HUMAN & KC1G3_HUMAN & $523(6)$ & $8(0)$ & 1 & 0 & 0 & $2(2)$ \\
\hline KPCA_HUMAN & JAK3_HUMAN & $427(14)$ & $6(3)$ & 0 & 1 & 0 & $1(1)$ \\
\hline KIT_HUMAN & D3DSX2_HUMAN & 0 & 0 & 1 & 0 & 0 & 0 \\
\hline KIT_HUMAN & RIPK2_HUMAN & $282(27)$ & $4(0)$ & 1 & 0 & 0 & 0 \\
\hline KIT_HUMAN & ABL2_HUMAN & $411(35)$ & $6(4)$ & 1 & 0 & 0 & $2(2)$ \\
\hline KDM6B_HUMAN & UTY_HUMAN & 0 & 0 & 1 & 0 & 0 & 0 \\
\hline KCC4_HUMAN & STK26_HUMAN & $265(7)$ & $2(0)$ & 1 & 0 & 0 & $2(2)$ \\
\hline KCC2D_HUMAN & VRK1_HUMAN & $93(0)$ & 0 & 0 & 0 & 1 & 0 \\
\hline KCC2D_HUMAN & STK26_HUMAN & $289(8)$ & 0 & 1 & 0 & 0 & 0 \\
\hline KC1G3_HUMAN & SRC_HUMAN & $508(5)$ & $6(0)$ & 1 & 0 & 0 & $2(2)$ \\
\hline KC1G3_HUMAN & CDK2_HUMAN & $572(4)$ & $11(2)$ & 1 & 0 & 6 & $2(1)$ \\
\hline KC1G3_HUMAN & ABL2_HUMAN & $531(4)$ & 0 & 1 & 0 & 0 & 0 \\
\hline KC1G3_HUMAN & KC1D_HUMAN & $292(14)$ & $2(2)$ & 1 & 0 & 0 & $2(2)$ \\
\hline KC1G3_HUMAN & CLK3_HUMAN & $551(6)$ & $2(0)$ & 1 & 0 & 0 & $2(2)$ \\
\hline KC1G3_HUMAN & SRPK2_HUMAN & $272(4)$ & $7(0)$ & 0 & 1 & 0 & $1(1)$ \\
\hline KC1G3_HUMAN & CDKL2_HUMAN & $88(5)$ & 0 & 1 & 0 & 0 & 0 \\
\hline KC1G3_HUMAN & SLK_HUMAN & $231(8)$ & $2(0)$ & 1 & 0 & 0 & $2(2)$ \\
\hline KC1G3_HUMAN & STK26_HUMAN & $275(6)$ & $2(0)$ & 1 & 0 & 0 & $2(2)$ \\
\hline KC1G1_HUMAN & SRC_HUMAN & $565(7)$ & $4(0)$ & 1 & 0 & 0 & $2(0)$ \\
\hline
\end{tabular}




\begin{tabular}{|c|c|c|c|c|c|c|c|}
\hline $\begin{array}{c}\text { UNIPROT NAME } \\
\text { Protein } 1\end{array}$ & $\begin{array}{c}\text { UNIPROTNAME } \\
\text { Protein } 2\end{array}$ & $\begin{array}{l}\text { Number of } \\
\text { reported duals } \\
\text { (actives) }^{a}\end{array}$ & $\begin{array}{c}\text { Number of reported } \\
\text { duals (similar to the } \\
\text { actives) }\end{array}$ & $\begin{array}{l}\text { known - } \\
\text { known }\end{array}$ & $\begin{array}{l}\text { known - } \\
\text { unknown }\end{array}$ & $\begin{array}{l}\text { unknown - } \\
\text { unknown }\end{array}$ & $\begin{array}{l}\text { Ligands with dual } \\
\text { activity (actives) }\end{array}$ \\
\hline KC1G1_HUMAN & MERTK_HUMAN & $428(8)$ & $2(0)$ & 1 & 0 & 0 & $2(0)$ \\
\hline KC1G1_HUMAN & KC1G3_HUMAN & $614(7)$ & $6(0)$ & 1 & 0 & 0 & $2(1)$ \\
\hline KC1E_HUMAN & KC1D_HUMAN & $419(238)$ & $2(2)$ & 1 & 0 & 0 & $2(2)$ \\
\hline KAPCA_HUMAN & AURKA_HUMAN & $669(9)$ & $4(0)$ & 1 & 0 & 0 & $2(0)$ \\
\hline KAPCA_HUMAN & ABL1_HUMAN & $648(12)$ & $4(0)$ & 1 & 0 & 0 & $2(2)$ \\
\hline KAPCA_HUMAN & AKT1_HUMAN & $768(35)$ & $104(0)$ & 1 & 0 & 0 & 0 \\
\hline KAPCA_HUMAN & AURKB_HUMAN & $622(9)$ & $4(0)$ & 1 & 0 & 0 & $2(1)$ \\
\hline JAK3_HUMAN & DAPK3_HUMAN & $302(20)$ & $4(0)$ & 1 & 0 & 0 & $2(0)$ \\
\hline JAK3_HUMAN & TYK2_HUMAN & $1185(622)$ & $14(14)$ & 2 & 0 & 0 & $4(4)$ \\
\hline JAK3_HUMAN & PKN1_HUMAN & $134(20)$ & $2(2)$ & 1 & 0 & 0 & $2(1)$ \\
\hline JAK2_HUMAN & DAPK3_HUMAN & $298(19)$ & $4(0)$ & 1 & 0 & 0 & $2(0)$ \\
\hline JAK2_HUMAN & TYK2_HUMAN & 1249 (789) & $306(236)$ & 4 & 4 & 0 & $12(12)$ \\
\hline JAK1_HUMAN & PKN1_HUMAN & $124(17)$ & $5(4)$ & 1 & 1 & 0 & $3(3)$ \\
\hline ITK_HUMAN & VGFR2_HUMAN & $616(21)$ & $8(4)$ & 1 & 0 & 0 & $2(2)$ \\
\hline HS90A_HUMAN & HS90B_HUMAN & $934(612)$ & $133(62)$ & 4 & 0 & 0 & $8(7)$ \\
\hline HS90A_HUMAN & ENPL_HUMAN & $172(40)$ & $80(30)$ & 1 & 0 & 0 & $2(1)$ \\
\hline HEXB_HUMAN & HNMT_HUMAN & 0 & 0 & 0 & 0 & 1 & 0 \\
\hline HEPS_HUMAN & UROK_HUMAN & $17(10)$ & $13(13)$ & 2 & 0 & 0 & $4(2)$ \\
\hline HDAC8_HUMAN & HDAC7_HUMAN & $2817(1630)$ & $2(2)$ & 1 & 0 & 0 & $2(2)$ \\
\hline HDAC8_HUMAN & HDAC2_HUMAN & 3208 (1899) & $720(402)$ & 1 & 0 & 0 & $2(2)$ \\
\hline HCK_HUMAN & LCK_HUMAN & $784(132)$ & $36(12)$ & 0 & 1 & 0 & 0 \\
\hline HCK_HUMAN & PIM1_HUMAN & $572(9)$ & 90 & 1 & 0 & 0 & $2(2)$ \\
\hline HCK_HUMAN & SRC_HUMAN & $955(290)$ & $348(286)$ & 0 & 1 & 0 & 0 \\
\hline HCK_HUMAN & PK3CG_HUMAN & $276(137)$ & $982(799)$ & 1 & 2 & 0 & $4(4)$ \\
\hline HCK_HUMAN & BMX_HUMAN & $564(23)$ & $28(6)$ & 0 & 0 & 1 & 0 \\
\hline HASP_HUMAN & KAPCA_HUMAN & 170 & $8(0)$ & 1 & 0 & 0 & $2(0)$ \\
\hline GSTA1_HUMAN & GSTP1_HUMAN & $25(9)$ & $18(16)$ & 1 & 0 & 0 & $2(2)$ \\
\hline GRM3_HUMAN & GRM7_HUMAN & $47(1)$ & $2(2)$ & 1 & 0 & 0 & $2(2)$ \\
\hline FTO_HUMAN & AK1BA_HUMAN & 0 & 0 & 0 & 0 & 1 & 0 \\
\hline FTO_HUMAN & THBG_HUMAN & 0 & 0 & 0 & 0 & 1 & 0 \\
\hline
\end{tabular}




\begin{tabular}{|c|c|c|c|c|c|c|c|}
\hline $\begin{array}{c}\text { UNIPROT NAME } \\
\text { Protein } 1\end{array}$ & $\begin{array}{c}\text { UNIPROTNAME } \\
\text { Protein } 2\end{array}$ & $\begin{array}{l}\text { Number of } \\
\text { reported duals } \\
\text { (actives) }^{a}\end{array}$ & $\begin{array}{c}\text { Number of reported } \\
\text { duals (similar to the } \\
\text { actives) }\end{array}$ & $\begin{array}{l}\text { known - } \\
\text { known }\end{array}$ & $\begin{array}{l}\text { known - } \\
\text { unknown }\end{array}$ & $\begin{array}{l}\text { unknown - } \\
\text { unknown }\end{array}$ & $\begin{array}{l}\text { Ligands with dual } \\
\text { activity (actives) }\end{array}$ \\
\hline FTO_HUMAN & ANDR_HUMAN & 0 & 0 & 0 & 1 & 0 & 0 \\
\hline FTO_HUMAN & AK1C3_HUMAN & 0 & 0 & 1 & 2 & 0 & 0 \\
\hline FOLR2_HUMAN & FOLR1_HUMAN & $25(12)$ & $4(4)$ & 1 & 0 & 0 & $2(2)$ \\
\hline FKBP5_HUMAN & FKB1A_HUMAN & $117(33)$ & $46(36)$ & 0 & 0 & 1 & 0 \\
\hline FGFR1_HUMAN & D3DSX2_HUMAN & 0 & 0 & 1 & 0 & 0 & 0 \\
\hline FAK1_HUMAN & FAK2_HUMAN & $531(78)$ & $66(59)$ & 1 & 0 & 0 & $2(2)$ \\
\hline FABPH_HUMAN & S10A7_HUMAN & 0 & 0 & 1 & 0 & 0 & 0 \\
\hline FABP5_HUMAN & FABP4_HUMAN & $103(66)$ & $6(6)$ & 1 & 0 & 0 & $2(2)$ \\
\hline FABP4_HUMAN & AK1C3_HUMAN & 0 & 0 & 2 & 0 & 0 & 0 \\
\hline FABP4_HUMAN & ACS2A_HUMAN & 0 & 0 & 1 & 1 & 0 & 0 \\
\hline FA7_HUMAN & UROK_HUMAN & $103(12)$ & $44(14)$ & 1 & 1 & 1 & 0 \\
\hline FA11_HUMAN & UROK_HUMAN & $57(4)$ & 0 & 0 & 0 & 1 & 0 \\
\hline ESR1_HUMAN & PPARG_HUMAN & $6(1)$ & 0 & 1 & 1 & 0 & 0 \\
\hline ERR3_HUMAN & ESR1_HUMAN & $21(19)$ & $38(34)$ & 1 & 0 & 0 & $2(2)$ \\
\hline ERR3_HUMAN & EST1_HUMAN & 0 & 0 & 0 & 0 & 1 & 0 \\
\hline ERR3_HUMAN & ESR2_HUMAN & $3(3)$ & $2(2)$ & 1 & 0 & 0 & $2(2)$ \\
\hline ERAP1_HUMAN & LKHA4_HUMAN & $1(0)$ & 0 & 1 & 0 & 0 & 0 \\
\hline EGLN1_HUMAN & FTO_HUMAN & $11(1)$ & $27(4)$ & 2 & 0 & 0 & $4(4)$ \\
\hline EGFR_HUMAN & TYK2_HUMAN & $211(27)$ & $13(8)$ & 0 & 5 & 0 & 0 \\
\hline DYRK2_HUMAN & PIM1_HUMAN & $599(36)$ & $2(2)$ & 1 & 0 & 0 & $2(2)$ \\
\hline DYR1A_HUMAN & GSK3B_HUMAN & $655(22)$ & $4(1)$ & 0 & 0 & 1 & 0 \\
\hline DYR1A_HUMAN & DYRK2_HUMAN & $515(47)$ & $2(2)$ & 1 & 0 & 0 & $2(2)$ \\
\hline DYR_HUMAN & FOLR2_HUMAN & $5(4)$ & $6(6)$ & 1 & 0 & 0 & $2(1)$ \\
\hline DDR1_HUMAN & D3DSX2_HUMAN & 0 & 0 & 1 & 0 & 0 & 0 \\
\hline DDR1_HUMAN & LCK_HUMAN & $128(47)$ & $6(4)$ & 1 & 0 & 0 & $2(2)$ \\
\hline DDR1_HUMAN & KIT_HUMAN & $189(77)$ & $28(16)$ & 2 & 0 & 0 & $4(4)$ \\
\hline DDR1_HUMAN & FGFR4_HUMAN & $108(20)$ & $6(2)$ & 1 & 0 & 0 & $2(1)$ \\
\hline DDR1_HUMAN & ABL2_HUMAN & $116(38)$ & $6(4)$ & 1 & 0 & 0 & $2(2)$ \\
\hline DDR1_HUMAN & MK14_HUMAN & $124(16)$ & $4(0)$ & 1 & 0 & 0 & $2(1)$ \\
\hline DCK_HUMAN & ATAD2_HUMAN & 0 & 0 & 0 & 0 & 3 & 0 \\
\hline
\end{tabular}




\begin{tabular}{|c|c|c|c|c|c|c|c|}
\hline $\begin{array}{c}\text { UNIPROT NAME } \\
\text { Protein } 1\end{array}$ & $\begin{array}{c}\text { UNIPROTNAME } \\
\text { Protein } 2\end{array}$ & $\begin{array}{l}\text { Number of } \\
\text { reported duals } \\
\text { (actives) }^{a}\end{array}$ & $\begin{array}{c}\text { Number of reported } \\
\text { duals (similar to the } \\
\text { actives) }\end{array}$ & $\begin{array}{l}\text { known - } \\
\text { known }\end{array}$ & $\begin{array}{l}\text { known - } \\
\text { unknown }\end{array}$ & $\begin{array}{l}\text { unknown - } \\
\text { unknown }\end{array}$ & $\begin{array}{l}\text { Ligands with dual } \\
\text { activity (actives) }\end{array}$ \\
\hline DAPK3_HUMAN & PIM1_HUMAN & $373(12)$ & $52(0)$ & 1 & 1 & 0 & 0 \\
\hline DAPK3_HUMAN & JAK1_HUMAN & $219(18)$ & $4(0)$ & 1 & 0 & 0 & $2(0)$ \\
\hline D3DSX2_HUMAN & FGFR4_HUMAN & 0 & 0 & 1 & 0 & 0 & 0 \\
\hline CSK21_HUMAN & CSK22_HUMAN & $1167(393)$ & $8(8)$ & 2 & 0 & 0 & $4(4)$ \\
\hline CSF1R_HUMAN & KIT_HUMAN & $626(141)$ & $108(0)$ & 0 & 0 & 1 & 0 \\
\hline CP46A_HUMAN & RORA_HUMAN & 0 & 0 & 1 & 0 & 0 & 0 \\
\hline CP3A4_HUMAN & BACE1_HUMAN & $17(4)$ & $1(1)$ & 0 & 0 & 1 & 0 \\
\hline CP2E1_HUMAN & CP2A6_HUMAN & $3(0)$ & $2(0)$ & 1 & 0 & 0 & $2(1)$ \\
\hline CP2E1_HUMAN & CP2AD_HUMAN & $1(0)$ & $2(0)$ & 1 & 0 & 0 & $2(1)$ \\
\hline CP2C9_HUMAN & AK1C2_HUMAN & 0 & 0 & 1 & 0 & 0 & 0 \\
\hline CP2AD_HUMAN & CP2A6_HUMAN & $5(3)$ & $2(2)$ & 1 & 0 & 0 & $2(2)$ \\
\hline CP11A_HUMAN & NPC1_HUMAN & 0 & 0 & 1 & 1 & 0 & 0 \\
\hline CP11A_HUMAN & ADRB2_HUMAN & 0 & 0 & 1 & 0 & 0 & 0 \\
\hline CP11A_HUMAN & RORA_HUMAN & 0 & 0 & 1 & 0 & 0 & 0 \\
\hline CLK3_HUMAN & KCC4_HUMAN & $434(5)$ & $2(0)$ & 1 & 0 & 0 & $2(2)$ \\
\hline CLK3_HUMAN & ABL2_HUMAN & $512(8)$ & 0 & 1 & 0 & 0 & 0 \\
\hline CLK3_HUMAN & CLK1_HUMAN & $307(58)$ & $2(2)$ & 1 & 0 & 0 & $2(2)$ \\
\hline CLK3_HUMAN & GSK3B_HUMAN & $580(12)$ & $3(1)$ & 0 & 0 & 1 & 0 \\
\hline CLK3_HUMAN & DYR1A_HUMAN & $443(49)$ & 0 & 1 & 0 & 0 & 0 \\
\hline CLK3_HUMAN & DYRK2_HUMAN & $475(11)$ & 0 & 1 & 0 & 0 & 0 \\
\hline CLK3_HUMAN & STK26_HUMAN & $274(6)$ & $2(0)$ & 1 & 0 & 0 & $2(2)$ \\
\hline CLK1_HUMAN & CHK1_HUMAN & $211(17)$ & $8(0)$ & 1 & 1 & 0 & $3(3)$ \\
\hline CLK1_HUMAN & ACK1_HUMAN & $165(15)$ & 0 & 1 & 0 & 0 & 0 \\
\hline CHK1_HUMAN & NEK2_HUMAN & $672(17)$ & $32(0)$ & 0 & 0 & 2 & 0 \\
\hline CHK1_HUMAN & BMP2K_HUMAN & $102(19)$ & $5(4)$ & 1 & 2 & 0 & $4(4)$ \\
\hline CDKL3_HUMAN & ST17B_HUMAN & $87(3)$ & $4(0)$ & 0 & 0 & 1 & 0 \\
\hline CDKL3_HUMAN & VRK1_HUMAN & $4(0)$ & 0 & 0 & 0 & 1 & 0 \\
\hline CDKL2_HUMAN & KCC4_HUMAN & $89(7)$ & 0 & 1 & 0 & 0 & 0 \\
\hline CDKL2_HUMAN & CLK3_HUMAN & $84(8)$ & 0 & 1 & 0 & 0 & 0 \\
\hline CDK9 HUMAN & BRD4_HUMAN & $9(1)$ & 0 & 1 & 0 & 0 & 0 \\
\hline
\end{tabular}




\begin{tabular}{|c|c|c|c|c|c|c|c|}
\hline $\begin{array}{l}\text { UNIPROT NAME } \\
\text { Protein } 1\end{array}$ & $\begin{array}{c}\text { UNIPROTNAME } \\
\text { Protein } 2\end{array}$ & $\begin{array}{l}\text { Number of } \\
\text { reported duals } \\
\text { (actives) }^{a}\end{array}$ & $\begin{array}{c}\text { Number of reported } \\
\text { duals (similar to the } \\
\text { actives) }\end{array}$ & $\begin{array}{l}\text { known - } \\
\text { known }\end{array}$ & $\begin{array}{l}\text { known - } \\
\text { unknown }\end{array}$ & $\begin{array}{l}\text { unknown - } \\
\text { unknown }\end{array}$ & $\begin{array}{l}\text { Ligands with dual } \\
\text { activity (actives) }\end{array}$ \\
\hline CDK9_HUMAN & CDK2_HUMAN & $720(281)$ & $528(470)$ & 3 & 0 & 0 & $6(6)$ \\
\hline CDK5_HUMAN & SRC_HUMAN & $615(10)$ & $9(2)$ & 1 & 0 & 0 & $2(2)$ \\
\hline CDK5_HUMAN & GSK3B_HUMAN & $1264(160)$ & $140(20)$ & 2 & 0 & 0 & $4(4)$ \\
\hline CDK5_HUMAN & KC1G3_HUMAN & $522(4)$ & $9(2)$ & 1 & 0 & 0 & $2(2)$ \\
\hline CDK2_HUMAN & TTHY_HUMAN & $5(0)$ & $2(0)$ & 1 & 0 & 0 & $2(2)$ \\
\hline CDK2_HUMAN & MYLK4_HUMAN & $99(6)$ & $4(0)$ & 0 & 0 & 1 & 0 \\
\hline CDK2_HUMAN & FABPH_HUMAN & 0 & 0 & 1 & 0 & 0 & 0 \\
\hline CDK2_HUMAN & ABL2_HUMAN & $564(8)$ & 0 & 0 & 1 & 0 & 0 \\
\hline CDK2_HUMAN & MAPK2_HUMAN & $875(21)$ & $18(2)$ & 1 & 0 & 0 & $2(2)$ \\
\hline CDK2_HUMAN & CLK1_HUMAN & $309(18)$ & $2(0)$ & 0 & 1 & 0 & $1(1)$ \\
\hline CDK2_HUMAN & NEK2_HUMAN & $749(8)$ & $25(0)$ & 0 & 1 & 0 & $1(0)$ \\
\hline CDK2_HUMAN & CDK5_HUMAN & $1100(244)$ & $421(349)$ & 3 & 0 & 0 & $6(2)$ \\
\hline CDK2_HUMAN & MERTK_HUMAN & $414(7)$ & 10 & 1 & 1 & 0 & $3(0)$ \\
\hline CDK2_HUMAN & TTBK1_HUMAN & $9(1)$ & $4(2)$ & 1 & 0 & 0 & $2(2)$ \\
\hline CDK2_HUMAN & KC1G1_HUMAN & $613(8)$ & $14(0)$ & 2 & 0 & 0 & $4(1)$ \\
\hline CD38_HUMAN & BST1_HUMAN & 0 & 0 & 1 & 0 & 0 & 0 \\
\hline CBR1_HUMAN & HCK_HUMAN & 0 & 0 & 0 & 0 & 1 & 0 \\
\hline CBR1_HUMAN & SRC_HUMAN & $2(0)$ & 0 & 0 & 0 & 1 & 0 \\
\hline CBR1_HUMAN & PK3CG_HUMAN & $1(0)$ & 0 & 0 & 0 & 1 & 0 \\
\hline CBR1_HUMAN & BMX_HUMAN & $1(0)$ & 0 & 0 & 0 & 1 & 0 \\
\hline CALRL_HUMAN & ST17B_HUMAN & 0 & 0 & 0 & 0 & 1 & 0 \\
\hline CAH9_HUMAN & CAH2_HUMAN & $3324(2258)$ & $218(216)$ & 1 & 0 & 0 & $2(2)$ \\
\hline CAH7_HUMAN & CAH1_HUMAN & $865(485)$ & $96(96)$ & 1 & 0 & 0 & $2(2)$ \\
\hline CAH7_HUMAN & MIF_HUMAN & $1(1)$ & 0 & 0 & 1 & 0 & 0 \\
\hline CAH7_HUMAN & CAH9_HUMAN & $530(294)$ & $50(50)$ & 1 & 0 & 0 & $2(2)$ \\
\hline CAH2_HUMAN & CAH7_HUMAN & $867(505)$ & 205 (189) & 4 & 0 & 0 & $8(8)$ \\
\hline CAH2_HUMAN & THBG_HUMAN & $1(1)$ & $2(2)$ & 1 & 0 & 0 & $2(2)$ \\
\hline CAH13_HUMAN & CAH2_HUMAN & 307 (186) & 971 (877) & 13 & 0 & 0 & $26(26)$ \\
\hline CAH13_HUMAN & CAH12_HUMAN & $276(157)$ & $266(240)$ & 4 & 0 & 0 & $8(8)$ \\
\hline CAH13 HUMAN & CAH1_HUMAN & $304(178)$ & $44(44)$ & 1 & 0 & 0 & $2(2)$ \\
\hline
\end{tabular}




\begin{tabular}{|c|c|c|c|c|c|c|c|}
\hline $\begin{array}{c}\text { UNIPROT NAME } \\
\text { Protein } 1\end{array}$ & $\begin{array}{c}\text { UNIPROTNAME } \\
\text { Protein } 2\end{array}$ & $\begin{array}{l}\text { Number of } \\
\text { reported duals } \\
\text { (actives) }^{a}\end{array}$ & $\begin{array}{c}\text { Number of reported } \\
\text { duals (similar to the } \\
\text { actives) }^{a}\end{array}$ & $\begin{array}{l}\text { known - } \\
\text { known }\end{array}$ & $\begin{array}{l}\text { known - } \\
\text { unknown }\end{array}$ & $\begin{array}{l}\text { unknown - } \\
\text { unknown }\end{array}$ & $\begin{array}{l}\text { Ligands with dual } \\
\text { activity (actives) }\end{array}$ \\
\hline CAH13_HUMAN & CAH7_HUMAN & $304(182)$ & $44(44)$ & 1 & 0 & 0 & $2(2)$ \\
\hline CAH13_HUMAN & CAH9_HUMAN & $212(101)$ & $44(44)$ & 1 & 0 & 0 & $2(2)$ \\
\hline CAH12_HUMAN & CAH2_HUMAN & $2721(1857)$ & $570(506)$ & 8 & 0 & 0 & $16(16)$ \\
\hline CAH12_HUMAN & CAH1_HUMAN & 2704 (1796) & $98(98)$ & 1 & 0 & 0 & $2(2)$ \\
\hline CAH12_HUMAN & CAH7_HUMAN & $637(358)$ & $48(48)$ & 1 & 0 & 0 & $2(2)$ \\
\hline CAH12_HUMAN & CAH9_HUMAN & $2520(2020)$ & $98(98)$ & 1 & 0 & 0 & $2(2)$ \\
\hline BTK_HUMAN & ABL1_HUMAN & $545(39)$ & $2(2)$ & 1 & 0 & 0 & $2(2)$ \\
\hline BTK_HUMAN & HCK_HUMAN & $494(30)$ & $14(6)$ & 1 & 0 & 0 & $2(2)$ \\
\hline BTK_HUMAN & BMX_HUMAN & $459(34)$ & $2(2)$ & 1 & 0 & 0 & $2(2)$ \\
\hline BRDT_HUMAN & BRD3_HUMAN & $56(12)$ & $2(2)$ & 1 & 0 & 0 & 0 \\
\hline BRD4_HUMAN & CBP_HUMAN & $436(136)$ & $60(24)$ & 1 & 1 & 0 & $1(1)$ \\
\hline BRD4_HUMAN & TNKS2_HUMAN & 0 & 0 & 0 & 0 & 2 & 0 \\
\hline BRD4_HUMAN & BRDT_HUMAN & $66(19)$ & $6(6)$ & 1 & 0 & 0 & $2(2)$ \\
\hline BRD4_HUMAN & BRD9_HUMAN & $118(26)$ & $28(12)$ & 2 & 1 & 0 & $5(5)$ \\
\hline BRD3_HUMAN & BRD4_HUMAN & 364 (269) & $6(6)$ & 1 & 0 & 0 & 0 \\
\hline BRD2_HUMAN & BRD3_HUMAN & $341(259)$ & $6(6)$ & 1 & 0 & 0 & 0 \\
\hline BRD2_HUMAN & BRDT_HUMAN & $56(12)$ & $2(2)$ & 1 & 0 & 0 & 0 \\
\hline BRAF_HUMAN & CDK8_HUMAN & $115(8)$ & $4(4)$ & 1 & 0 & 0 & $2(2)$ \\
\hline BMX_HUMAN & LCK_HUMAN & $579(38)$ & $22(6)$ & 1 & 0 & 0 & 0 \\
\hline BMX_HUMAN & RET_HUMAN & $537(24)$ & $23(4)$ & 0 & 0 & 1 & 0 \\
\hline BMX_HUMAN & SRC_HUMAN & $567(32)$ & $18(6)$ & 0 & 0 & 1 & 0 \\
\hline BMX_HUMAN & MK14_HUMAN & $567(5)$ & $2(2)$ & 1 & 0 & 0 & $2(2)$ \\
\hline BACE1_HUMAN & BACE2_HUMAN & $1317(1054)$ & $146(144)$ & 1 & 0 & 0 & $2(2)$ \\
\hline AVR2A_HUMAN & ULK1_HUMAN & $89(4)$ & $2(0)$ & 0 & 0 & 1 & 0 \\
\hline AVR2A_HUMAN & AAPK2_HUMAN & $104(5)$ & 0 & 1 & 0 & 0 & 0 \\
\hline AVR2A_HUMAN & ACVR1_HUMAN & $105(8)$ & 0 & 1 & 0 & 0 & 0 \\
\hline AVR2A_HUMAN & KCC2D_HUMAN & $99(4)$ & 0 & 1 & 0 & 0 & 0 \\
\hline AVR2A_HUMAN & STK26_HUMAN & $101(8)$ & 0 & 1 & 0 & 0 & 0 \\
\hline AURKB_HUMAN & ABL2_HUMAN & $603(26)$ & $4(4)$ & 1 & 0 & 0 & $2(2)$ \\
\hline AURKA_HUMAN & ITK HUMAN & $632(37)$ & $10(10)$ & 1 & 0 & 0 & $2(2)$ \\
\hline
\end{tabular}




\begin{tabular}{|c|c|c|c|c|c|c|c|}
\hline $\begin{array}{c}\text { UNIPROT NAME } \\
\text { Protein } 1\end{array}$ & $\begin{array}{c}\text { UNIPROTNAME } \\
\text { Protein } 2\end{array}$ & $\begin{array}{l}\text { Number of } \\
\text { reported duals } \\
\text { (actives) }^{a}\end{array}$ & $\begin{array}{c}\text { Number of reported } \\
\text { duals (similar to the } \\
\text { actives) }\end{array}$ & $\begin{array}{l}\text { known - } \\
\text { known }\end{array}$ & $\begin{array}{l}\text { known - } \\
\text { unknown }\end{array}$ & $\begin{array}{l}\text { unknown - } \\
\text { unknown }\end{array}$ & $\begin{array}{l}\text { Ligands with dual } \\
\text { activity (actives) }\end{array}$ \\
\hline ATS5_HUMAN & MMP8_HUMAN & $24(16)$ & $2(2)$ & 1 & 0 & 0 & 0 \\
\hline ATS5_HUMAN & MMP12_HUMAN & $26(12)$ & $2(2)$ & 1 & 0 & 0 & 0 \\
\hline ATS1_HUMAN & ATS5_HUMAN & $14(5)$ & 0 & 1 & 0 & 0 & 0 \\
\hline ANDR_HUMAN & TTHY_HUMAN & $8(1)$ & $6(0)$ & 1 & 0 & 0 & $2(2)$ \\
\hline ANDR_HUMAN & AK1BA_HUMAN & $3(0)$ & $2(0)$ & 1 & 0 & 0 & $2(2)$ \\
\hline ANDR_HUMAN & ALBU_HUMAN & $6(0)$ & 0 & 1 & 0 & 0 & 0 \\
\hline ANDR_HUMAN & THBG_HUMAN & $1(0)$ & 0 & 0 & 0 & 1 & 0 \\
\hline ANDR_HUMAN & HCK_HUMAN & 0 & 0 & 0 & 0 & 1 & 0 \\
\hline ANDR_HUMAN & THB_HUMAN & $18(3)$ & $4(0)$ & 1 & 0 & 0 & $2(0)$ \\
\hline ANDR_HUMAN & PTGIS_HUMAN & 0 & 0 & 1 & 0 & 0 & 0 \\
\hline AMYP_HUMAN & TTHY_HUMAN & $1(0)$ & $4(0)$ & 1 & 1 & 0 & $3(3)$ \\
\hline ALK_HUMAN & ROS1_HUMAN & $634(59)$ & $4(2)$ & 1 & 0 & 0 & $2(2)$ \\
\hline ALDR_HUMAN & AK1C2_HUMAN & $19(1)$ & 0 & 0 & 0 & 1 & 0 \\
\hline ALBU_HUMAN & PPARG_HUMAN & $8(2)$ & $2(0)$ & 1 & 0 & 0 & $2(0)$ \\
\hline ALBU_HUMAN & FABP4_HUMAN & $2(1)$ & 0 & 1 & 1 & 0 & 0 \\
\hline ALBU_HUMAN & ACS2A_HUMAN & 0 & 0 & 1 & 0 & 0 & 0 \\
\hline AL1L1_HUMAN & NOS2_HUMAN & 0 & 0 & 0 & 0 & 2 & 0 \\
\hline AKT2_HUMAN & AKT1_HUMAN & $1589(693)$ & $59(57)$ & 1 & 0 & 0 & $2(2)$ \\
\hline AK1C3_HUMAN & TTHY_HUMAN & $8(1)$ & $49(10)$ & 4 & 3 & 0 & $8(8)$ \\
\hline AK1C3_HUMAN & CP2C9_HUMAN & $1(0)$ & 0 & 0 & 0 & 1 & 0 \\
\hline AK1C3_HUMAN & ALDR_HUMAN & $20(2)$ & 0 & 0 & 0 & 1 & 0 \\
\hline AK1C3_HUMAN & PPARG_HUMAN & $2(0)$ & $4(0)$ & 1 & 1 & 1 & $3(1)$ \\
\hline AK1C3_HUMAN & NMRL1_HUMAN & 0 & 0 & 0 & 0 & 1 & 0 \\
\hline AK1C3_HUMAN & ALBU_HUMAN & $7(0)$ & $8(0)$ & 1 & 2 & 0 & $3(0)$ \\
\hline AK1C3_HUMAN & THBG_HUMAN & $1(1)$ & $6(6)$ & 1 & 2 & 0 & $4(4)$ \\
\hline AK1C3_HUMAN & PTGR2_HUMAN & 0 & 0 & 1 & 1 & 0 & 0 \\
\hline AK1C2_HUMAN & TTHY_HUMAN & $6(1)$ & $6(2)$ & 2 & 0 & 0 & $2(1)$ \\
\hline AK1C2_HUMAN & AK1C3_HUMAN & $387(89)$ & $1096(504)$ & 7 & 1 & 1 & $13(11)$ \\
\hline AK1C2_HUMAN & AK1BA_HUMAN & $17(3)$ & $24(4)$ & 1 & 0 & 0 & $2(2)$ \\
\hline AK1C2 HUMAN & ALBU_HUMAN & $4(0)$ & $2(0)$ & 1 & 0 & 0 & $2(0)$ \\
\hline
\end{tabular}




\begin{tabular}{|c|c|c|c|c|c|c|c|}
\hline $\begin{array}{l}\text { UNIPROT NAME } \\
\text { Protein } 1\end{array}$ & $\begin{array}{l}\text { UNIPROTNAME } \\
\text { Protein } 2\end{array}$ & $\begin{array}{l}\text { Number of } \\
\text { reported duals } \\
\text { (actives) }^{a}\end{array}$ & $\begin{array}{c}\text { Number of reported } \\
\text { duals (similar to the } \\
\text { actives) }\end{array}$ & $\begin{array}{l}\text { known - } \\
\text { known }\end{array}$ & $\begin{array}{l}\text { known - } \\
\text { unknown }\end{array}$ & $\begin{array}{l}\text { unknown - } \\
\text { unknown }\end{array}$ & $\begin{array}{l}\text { Ligands with dual } \\
\text { activity (actives) }\end{array}$ \\
\hline AK1C2_HUMAN & ANDR_HUMAN & $8(4)$ & $6(0)$ & 0 & 1 & 0 & $1(0)$ \\
\hline AK1C2_HUMAN & FTO_HUMAN & 0 & 0 & 0 & 1 & 0 & 0 \\
\hline AK1BA_HUMAN & NMRL1_HUMAN & 0 & 0 & 0 & 0 & 1 & 0 \\
\hline AK1BA_HUMAN & THBG_HUMAN & $1(1)$ & $2(2)$ & 0 & 1 & 0 & $1(1)$ \\
\hline AK1BA_HUMAN & ALDR_HUMAN & $103(55)$ & $26(20)$ & 5 & 2 & 0 & $11(4)$ \\
\hline AK1BA_HUMAN & AK1C3_HUMAN & $17(3)$ & $72(12)$ & 2 & 1 & 0 & $5(5)$ \\
\hline ADRB2_HUMAN & RORA_HUMAN & 0 & 0 & 1 & 0 & 0 & 0 \\
\hline ADRB2_HUMAN & RORG_HUMAN & 0 & 0 & 0 & 0 & 1 & 0 \\
\hline ADRB2_HUMAN & NR1H2_HUMAN & 0 & 0 & 0 & 0 & 1 & 0 \\
\hline ACVR1_HUMAN & AAPK2_HUMAN & $109(13)$ & $4(4)$ & 1 & 0 & 0 & $2(2)$ \\
\hline ACVL1_HUMAN & BMR1B_HUMAN & $99(20)$ & $18(14)$ & 1 & 0 & 0 & $2(2)$ \\
\hline ACVL1_HUMAN & ACVR1_HUMAN & $123(23)$ & $20(16)$ & 1 & 0 & 0 & $2(2)$ \\
\hline ACS2A_HUMAN & AK1C3_HUMAN & 0 & 0 & 0 & 1 & 0 & 0 \\
\hline ACE_HUMAN & LKHA4_HUMAN & $2(1)$ & $4(4)$ & 1 & 1 & 0 & $3(1)$ \\
\hline ABL2_HUMAN & KCC4_HUMAN & $443(6)$ & 0 & 1 & 0 & 0 & 0 \\
\hline ABL2_HUMAN & AURKA_HUMAN & $622(21)$ & $4(4)$ & 1 & 0 & 0 & $2(2)$ \\
\hline ABL2_HUMAN & ABL1_HUMAN & 789 (199) & $34(27)$ & 2 & 1 & 0 & $5(5)$ \\
\hline ABL2_HUMAN & KAPCA_HUMAN & $555(6)$ & $4(0)$ & 1 & 0 & 0 & $2(0)$ \\
\hline ABL2_HUMAN & MK14_HUMAN & $626(16)$ & $4(0)$ & 1 & 0 & 0 & $2(1)$ \\
\hline ABL2_HUMAN & CDKL2_HUMAN & $89(15)$ & 0 & 1 & 0 & 0 & 0 \\
\hline ABL2_HUMAN & SLK_HUMAN & $169(34)$ & 0 & 1 & 0 & 0 & 0 \\
\hline ABL2_HUMAN & STK26_HUMAN & $284(16)$ & 0 & 1 & 0 & 0 & 0 \\
\hline ABL1_HUMAN & STK10_HUMAN & $520(48)$ & $5(5)$ & 1 & 1 & 0 & $3(2)$ \\
\hline ABL1_HUMAN & LCK_HUMAN & $1019(124)$ & $12(8)$ & 1 & 1 & 0 & $3(1)$ \\
\hline ABL1_HUMAN & BMX_HUMAN & $517(29)$ & $2(2)$ & 1 & 0 & 0 & $2(2)$ \\
\hline ABL1_HUMAN & DDR1_HUMAN & $149(50)$ & $12(8)$ & 1 & 1 & 0 & $3(3)$ \\
\hline 8ODP_HUMAN & HS90A_HUMAN & 0 & 0 & 0 & 0 & 3 & 0 \\
\hline 1433Z HUMAN & 1433S HUMAN & 0 & 0 & 0 & 0 & 1 & 0 \\
\hline
\end{tabular}

${ }^{a}$ Ligands whom activity annotations were reported in the same unit $\left(\mathrm{IC}_{50}, \mathrm{~K}_{\mathrm{i}}, \mathrm{K}_{\mathrm{d}}\right.$ or $\left.\mathrm{EC}_{50}\right)$, with values below $5 \mu \mathrm{M}$ for the two targets under study.

${ }^{\mathrm{b}}$ Ligands whom scaffold was different from those already reported in the literature. 
Table S3: Percentage of sequence identity and sequence similarity of the identified target associations. Records are sorted according to the evaluated percentages of sequence similarity.

\begin{tabular}{|c|c|c|c|c|}
\hline $\begin{array}{c}\text { UNIPROT NAME } \\
\text { Protein } 1 \\
\end{array}$ & $\begin{array}{c}\text { UNIPROT NAME } \\
\text { Protein } 2 \\
\end{array}$ & TTD & $\begin{array}{c}\% \text { of sequence } \\
\text { similarity }\end{array}$ & $\begin{array}{c}\% \text { of sequence } \\
\text { identity }\end{array}$ \\
\hline FGFR1_HUMAN & D3DSX2_HUMAN & $\mathrm{NO}$ & 100 & 100 \\
\hline CP2AD_HUMAN & CP2A6_HUMAN & NO & 96.6 & 93.5 \\
\hline HS90A_HUMAN & HS90B_HUMAN & NO & 93.4 & 85.8 \\
\hline AKT2_HUMAN & AKT1_HUMAN & NO & 92.5 & 81.6 \\
\hline AK1C2_HUMAN & AK1C3_HUMAN & NO & 92 & 87 \\
\hline TNKS1_HUMAN & TNKS2_HUMAN & NO & 90.7 & 82.2 \\
\hline KC1E_HUMAN & KC1D_HUMAN & NO & 89.7 & 85 \\
\hline MK03_HUMAN & MK01_HUMAN & NO & 89.3 & 83.7 \\
\hline MK11_HUMAN & MK14_HUMAN & NO & 88.9 & 75.5 \\
\hline CSK21_HUMAN & CSK22_HUMAN & NO & 88.9 & 82.1 \\
\hline PPIF_HUMAN & PPIA_HUMAN & $\mathrm{NO}$ & 85.8 & 76.5 \\
\hline RARB_HUMAN & RARA_HUMAN & NO & 85.3 & 77.8 \\
\hline D3DSX2_HUMAN & FGFR4_HUMAN & NO & 84.7 & 73.1 \\
\hline PDE4B_HUMAN & PDE4D_HUMAN & YES & 84.6 & 75.9 \\
\hline KC1G1_HUMAN & KC1G3_HUMAN & NO & 83.8 & 77.2 \\
\hline NMNA3_HUMAN & NRK1_HUMAN & NO & 83.3 & 50 \\
\hline CAH7_HUMAN & MIF_HUMAN & NO & 83.3 & 83.3 \\
\hline 1433Z_HUMAN & 1433S_HUMAN & NO & 83.3 & 71.1 \\
\hline AK1BA_HUMAN & ALDR_HUMAN & NO & 83.2 & 70.6 \\
\hline PPARA_HUMAN & PPARD_HUMAN & YES & 82.9 & 68.8 \\
\hline GRM3_HUMAN & GRM2_HUMAN & YES & 82.8 & 68.7 \\
\hline FGFR2_HUMAN & FGFR1_HUMAN & YES & 82.6 & 71.7 \\
\hline PPARG_HUMAN & PPARD_HUMAN & YES & 80.6 & 62.9 \\
\hline FOLR2_HUMAN & FOLR1_HUMAN & NO & 80.5 & 73.2 \\
\hline MAPK2_HUMAN & MAPK3_HUMAN & NO & 80.2 & 67.4 \\
\hline CDK2_HUMAN & TTHY_HUMAN & NO & 80 & 50 \\
\hline UPP1_HUMAN & UPP2_HUMAN & NO & 79.9 & 66.1 \\
\hline PDE4B_HUMAN & PDE4A_HUMAN & YES & 79.4 & 71.1 \\
\hline HCK_HUMAN & LCK_HUMAN & NO & 78.4 & 65.4 \\
\hline LCK_HUMAN & SRC_HUMAN & NO & 77.8 & 59.9 \\
\hline CAH13_HUMAN & CAH1_HUMAN & NO & 77.4 & 59.8 \\
\hline PDE4A_HUMAN & PDE4D_HUMAN & YES & 75.6 & 64.2 \\
\hline CAH13_HUMAN & CAH2_HUMAN & NO & 75 & 60.5 \\
\hline HCK_HUMAN & SRC_HUMAN & NO & 74.9 & 58.2 \\
\hline CDK2_HUMAN & CDK5_HUMAN & NO & 74.5 & 59.9 \\
\hline NOS1_HUMAN & NOS3_HUMAN & NO & 74.1 & 59 \\
\hline MMP8_HUMAN & MMP1_HUMAN & NO & 74.1 & 58 \\
\hline FGFR4_HUMAN & FGFR1_HUMAN & YES & 73.4 & 58.5 \\
\hline AURKB_HUMAN & AURKA_HUMAN & YES & 73.2 & 61.8 \\
\hline NR1H2_HUMAN & NR1H3_HUMAN & NO & 72.7 & 62.5 \\
\hline CAH13_HUMAN & CAH7_HUMAN & NO & 72.7 & 53.9 \\
\hline CAH2_HUMAN & CAH1_HUMAN & YES & 72.6 & 60.2 \\
\hline ACVL1_HUMAN & ACVR1_HUMAN & NO & 72.4 & 60.5 \\
\hline PIM1_HUMAN & PIM2_HUMAN & NO & 72 & 58.4 \\
\hline FABP5_HUMAN & FABP4_HUMAN & NO & 72 & 55.2 \\
\hline TTHY_HUMAN & FABPH_HUMAN & NO & 71.4 & 38.1 \\
\hline CAH2_HUMAN & CAH7_HUMAN & NO & 71.4 & 56.4 \\
\hline BRD2_HUMAN & BRD3_HUMAN & NO & 71.1 & 62.6 \\
\hline PH4H_HUMAN & TPH1_HUMAN & NO & 69.5 & 55.2 \\
\hline CAH7_HUMAN & CAH1_HUMAN & NO & 69.5 & 51.6 \\
\hline JAK2_HUMAN & JAK3_HUMAN & YES & 69 & 50.5 \\
\hline
\end{tabular}




\begin{tabular}{|c|c|c|c|c|}
\hline AK1C2_HUMAN & TTHY_HUMAN & NO & 68.8 & 37.5 \\
\hline MMP3_HUMAN & MMP12_HUMAN & NO & 68.7 & 51.4 \\
\hline PPARG_HUMAN & PPARA_HUMAN & YES & 68.4 & 54.7 \\
\hline MK14_HUMAN & MK01_HUMAN & YES & 68.3 & 49 \\
\hline BRAF_HUMAN & RAF1_HUMAN & YES & 68.3 & 55.9 \\
\hline ALDR_HUMAN & AK1C2_HUMAN & NO & 68.2 & 49 \\
\hline NOS2_HUMAN & NOS1_HUMAN & NO & 67.9 & 53.6 \\
\hline AK1C3_HUMAN & ALDR_HUMAN & NO & 67.6 & 48.3 \\
\hline CP2E1_HUMAN & CP2A6_HUMAN & NO & 67.2 & 47.6 \\
\hline HDAC8_HUMAN & HDAC2_HUMAN & NO & 66.6 & 43.4 \\
\hline CP2E1_HUMAN & CP2AD_HUMAN & NO & 66.6 & 47.6 \\
\hline BRD3_HUMAN & BRD4_HUMAN & NO & 66.4 & 57.6 \\
\hline AK1C2_HUMAN & AK1BA_HUMAN & NO & 66.1 & 48.7 \\
\hline MMP8_HUMAN & MMP13_HUMAN & NO & 65.4 & 50.1 \\
\hline BRD2_HUMAN & BRDT_HUMAN & NO & 65.4 & 51.3 \\
\hline BTK_HUMAN & BMX_HUMAN & NO & 65.3 & 47.7 \\
\hline HS90A_HUMAN & ENPL_HUMAN & NO & 64.9 & 46.4 \\
\hline MK14_HUMAN & MK09_HUMAN & YES & 64.8 & 49.7 \\
\hline MMP8_HUMAN & MMP12_HUMAN & NO & 64.8 & 47.7 \\
\hline NOS3_HUMAN & NOS2_HUMAN & YES & 64.6 & 50 \\
\hline AK1BA_HUMAN & AK1C3_HUMAN & NO & 64.6 & 47.5 \\
\hline RORA_HUMAN & RORG_HUMAN & NO & 64.3 & 49.7 \\
\hline KC1G3_HUMAN & KC1D_HUMAN & NO & 64.3 & 47.6 \\
\hline MMP1_HUMAN & MMP13_HUMAN & NO & 64.2 & 48.1 \\
\hline FKBP5_HUMAN & FKB1A_HUMAN & NO & 64.2 & 51.6 \\
\hline CLK3_HUMAN & CLK1_HUMAN & NO & 64.2 & 49.9 \\
\hline BRD4_HUMAN & BRD2_HUMAN & YES & 63.8 & 52.8 \\
\hline EGFR_HUMAN & ERBB2_HUMAN & YES & 63.7 & 49.7 \\
\hline ACVL1_HUMAN & BMR1B_HUMAN & NO & 63.5 & 45.5 \\
\hline ABL1_HUMAN & SRC_HUMAN & YES & 63.4 & 44.2 \\
\hline ESR2_HUMAN & ESR1_HUMAN & YES & 63.3 & 47.1 \\
\hline TYK2_HUMAN & JAK1_HUMAN & NO & 63.3 & 46.4 \\
\hline BRDT_HUMAN & BRD3_HUMAN & NO & 63.1 & 51.6 \\
\hline BACE1_HUMAN & BACE2_HUMAN & NO & 62.7 & 47.4 \\
\hline ABL2_HUMAN & ABL1_HUMAN & NO & 62.7 & 53 \\
\hline ABL1_HUMAN & LCK_HUMAN & NO & 62.4 & 43.4 \\
\hline LCK_HUMAN & ABL2_HUMAN & NO & 62.3 & 44.1 \\
\hline JAK2_HUMAN & JAK1_HUMAN & YES & 61.5 & 44 \\
\hline PAK5_HUMAN & PAK4_HUMAN & NO & 61.4 & 52.4 \\
\hline FAK1_HUMAN & FAK2_HUMAN & NO & 60.9 & 44.5 \\
\hline GRM3_HUMAN & GRM7_HUMAN & NO & 60.8 & 44.6 \\
\hline HBA_HUMAN & HBB_HUMAN & YES & 60.7 & 43.4 \\
\hline ALK_HUMAN & ROS1_HUMAN & NO & 60 & 39 \\
\hline QPCTL_HUMAN & QPCT_HUMAN & NO & 59.9 & 46.8 \\
\hline KAPCA_HUMAN & AKT1_HUMAN & NO & 59.8 & 42.9 \\
\hline PARP2_HUMAN & PARP1_HUMAN & NO & 59.7 & 41 \\
\hline PAK6_HUMAN & PAK4_HUMAN & NO & 58.4 & 48.2 \\
\hline BTK_HUMAN & ABL1_HUMAN & NO & 58.1 & 39.1 \\
\hline STK25_HUMAN & PAK6_HUMAN & NO & 57.9 & 38.2 \\
\hline GSK3B_HUMAN & CDK2_HUMAN & YES & 57.7 & 33.6 \\
\hline RET_HUMAN & LCK_HUMAN & NO & 57.5 & 39.5 \\
\hline NEP_HUMAN & ECE1_HUMAN & NO & 57.5 & 39 \\
\hline CAH13_HUMAN & CAH12_HUMAN & NO & 57 & 37.3 \\
\hline CDK9_HUMAN & CDK2_HUMAN & NO & 56.9 & 39.1 \\
\hline BTK_HUMAN & HCK_HUMAN & NO & 56.5 & 36.4 \\
\hline KIT_HUMAN & D3DSX2_HUMAN & NO & 56.2 & 40.3 \\
\hline
\end{tabular}




\begin{tabular}{|c|c|c|c|c|}
\hline CSF1R_HUMAN & KIT_HUMAN & NO & 55.9 & 40.9 \\
\hline JAK3_HUMAN & JAK1_HUMAN & YES & 55.8 & 39.7 \\
\hline PLK2_HUMAN & PLK1_HUMAN & YES & 55.7 & 38 \\
\hline RET_HUMAN & HCK_HUMAN & NO & 55.7 & 37.6 \\
\hline SLK_HUMAN & STK26_HUMAN & NO & 55.4 & 34.5 \\
\hline CAH12_HUMAN & CAH7_HUMAN & NO & 55.1 & 39.2 \\
\hline ABL1_HUMAN & BMX_HUMAN & NO & 55 & 34.6 \\
\hline MK14_HUMAN & CDK2_HUMAN & YES & 54.3 & 38.1 \\
\hline JAK2_HUMAN & TYK2_HUMAN & NO & 54.3 & 38 \\
\hline DYR1A_HUMAN & DYRK2_HUMAN & NO & 54.2 & 39 \\
\hline ERBB3_HUMAN & SRC_HUMAN & YES & 54.1 & 37.1 \\
\hline KDM6B_HUMAN & UTY_HUMAN & NO & 54.1 & 41.3 \\
\hline CDK5_HUMAN & GSK3B_HUMAN & NO & 54.1 & 34.9 \\
\hline ATS1_HUMAN & ATS5_HUMAN & NO & 54.1 & 41.5 \\
\hline SRC_HUMAN & RET_HUMAN & YES & 53.8 & 37.2 \\
\hline ERR3_HUMAN & ESR2_HUMAN & NO & 53.8 & 35.1 \\
\hline KAPCA_HUMAN & AURKB_HUMAN & NO & 53.7 & 31.7 \\
\hline CD38_HUMAN & BST1_HUMAN & NO & 53.7 & 36.8 \\
\hline CAH12_HUMAN & CAH1_HUMAN & NO & 53.6 & 36.1 \\
\hline PDE2A_HUMAN & PDE9A_HUMAN & NO & 53.2 & 31.2 \\
\hline HEPS_HUMAN & UROK_HUMAN & NO & 53.1 & 34.4 \\
\hline BMX_HUMAN & LCK_HUMAN & NO & 53.1 & 34.8 \\
\hline MMP8_HUMAN & MMP16_HUMAN & NO & 53 & 37 \\
\hline JAK3_HUMAN & TYK2_HUMAN & NO & 52.8 & 39 \\
\hline CAH7_HUMAN & CAH9_HUMAN & NO & 52.8 & 37.5 \\
\hline ERR3_HUMAN & ESR1_HUMAN & NO & 52.6 & 36.7 \\
\hline PDE8A_HUMAN & PDE9A_HUMAN & NO & 52.5 & 33.4 \\
\hline BMX_HUMAN & SRC_HUMAN & NO & 52.5 & 35.6 \\
\hline PDE7A_HUMAN & PDE8A_HUMAN & NO & 52.2 & 31.6 \\
\hline KAPCA_HUMAN & AURKA_HUMAN & NO & 52.1 & 31.1 \\
\hline GSTA1_HUMAN & GSTP1_HUMAN & NO & 52 & 31.8 \\
\hline FA11_HUMAN & UROK_HUMAN & NO & 51.9 & 33.5 \\
\hline CAH12_HUMAN & CAH2_HUMAN & NO & 51.9 & 35.6 \\
\hline AVR2A_HUMAN & ACVR1_HUMAN & NO & 51.6 & 31.3 \\
\hline ADRB2_HUMAN & RORG_HUMAN & NO & 51.5 & 39.4 \\
\hline NQO2_HUMAN & ABL2_HUMAN & NO & 51.4 & 21.6 \\
\hline DDR1_HUMAN & D3DSX2_HUMAN & NO & 51.4 & 35.9 \\
\hline PDE7A_HUMAN & PDE9A_HUMAN & NO & 51.3 & 31.8 \\
\hline CAH13_HUMAN & CAH9_HUMAN & NO & 51.3 & 36.4 \\
\hline MDM2_HUMAN & MDM4_HUMAN & NO & 51.2 & 31.7 \\
\hline HCK_HUMAN & BMX_HUMAN & NO & 51.2 & 31.8 \\
\hline ABL1_HUMAN & KSYK_HUMAN & YES & 51 & 33.8 \\
\hline MK01_HUMAN & GSK3B_HUMAN & YES & 50.9 & 32.8 \\
\hline MMP12_HUMAN & MMP16_HUMAN & NO & 50.8 & 34.6 \\
\hline CAH9_HUMAN & CAH2_HUMAN & NO & 50.6 & 34.5 \\
\hline PAK1_HUMAN & PAK4_HUMAN & NO & 50.5 & 36.7 \\
\hline ABL1_HUMAN & DDR1_HUMAN & NO & 50.4 & 36.1 \\
\hline PDE7A_HUMAN & PDE4D_HUMAN & NO & 50.3 & 29.8 \\
\hline ABL2_HUMAN & MK14_HUMAN & NO & 50.2 & 28.9 \\
\hline PK3CA_HUMAN & PK3CG_HUMAN & YES & 50.1 & 33.6 \\
\hline MK14_HUMAN & LKHA4_HUMAN & YES & 50 & 30 \\
\hline TTHY_HUMAN & S10A7_HUMAN & NO & 50 & 22.7 \\
\hline FABPH_HUMAN & S10A7_HUMAN & NO & 50 & 30.8 \\
\hline PDE7A_HUMAN & PDE5A_HUMAN & NO & 49.8 & 29.9 \\
\hline CAH1_HUMAN & CAH9_HUMAN & YES & 49.4 & 32.6 \\
\hline LCK_HUMAN & MK14_HUMAN & NO & 49.2 & 30.3 \\
\hline
\end{tabular}




\begin{tabular}{|c|c|c|c|c|}
\hline CDK2_HUMAN & FABPH_HUMAN & NO & 48.8 & 29.3 \\
\hline CAH4_HUMAN & CAH2_HUMAN & YES & 48.7 & 34.9 \\
\hline CDK2_HUMAN & SRC_HUMAN & YES & 48.6 & 26.3 \\
\hline TYK2_HUMAN & DAPK3_HUMAN & NO & 48.6 & 31.3 \\
\hline CAH12_HUMAN & CAH9_HUMAN & NO & 48.6 & 35.8 \\
\hline JAK2_HUMAN & DAPK3_HUMAN & NO & 48.5 & 29.8 \\
\hline AURKA_HUMAN & CHK1_HUMAN & YES & 48.4 & 28.5 \\
\hline ULK1_HUMAN & KCC2D_HUMAN & NO & 48.4 & 32.6 \\
\hline S10A7_HUMAN & CDK2_HUMAN & NO & 48.4 & 33.9 \\
\hline AURKA_HUMAN & CDK2_HUMAN & YES & 48 & 29.5 \\
\hline XIAP_HUMAN & BIRC2_HUMAN & NO & 47.9 & 34 \\
\hline ALBU_HUMAN & FABP4_HUMAN & NO & 47.8 & 30.4 \\
\hline BMX_HUMAN & MK14_HUMAN & NO & 47.7 & 26 \\
\hline TTHY_HUMAN & AK1BA_HUMAN & NO & 47.6 & 33.3 \\
\hline BMX_HUMAN & RET_HUMAN & NO & 47.6 & 31 \\
\hline ABL2_HUMAN & KAPCA_HUMAN & NO & 47.6 & 28 \\
\hline PIM1_HUMAN & CHK2_HUMAN & YES & 47.5 & 30.2 \\
\hline MK14_HUMAN & BRAF_HUMAN & YES & 47.3 & 26.9 \\
\hline GCR_HUMAN & PRGR_HUMAN & YES & 47.2 & 33.4 \\
\hline MRCKB_HUMAN & ROCK1_HUMAN & NO & 47.1 & 27.9 \\
\hline FA7_HUMAN & FA11_HUMAN & YES & 46.9 & 31.1 \\
\hline ESR1_HUMAN & MIF_HUMAN & YES & 46.9 & 24.5 \\
\hline DAPK3_HUMAN & JAK1_HUMAN & NO & 46.9 & 27.8 \\
\hline FAK1_HUMAN & CDK2_HUMAN & YES & 46.8 & 26.2 \\
\hline BTK_HUMAN & MK14_HUMAN & YES & 46.8 & 26.1 \\
\hline STK25_HUMAN & KCC1G_HUMAN & NO & 46.8 & 27.5 \\
\hline CDK2_HUMAN & ABL2_HUMAN & NO & 46.7 & 28.2 \\
\hline 8ODP_HUMAN & HS90A_HUMAN & NO & 46.7 & 31.1 \\
\hline DDR1_HUMAN & ABL2_HUMAN & NO & 46.6 & 32 \\
\hline KS6A1_HUMAN & CDK2_HUMAN & NO & 46.4 & 29.1 \\
\hline FAK1_HUMAN & MK14_HUMAN & YES & 46.3 & 26.9 \\
\hline S10A4_HUMAN & TNNC1_HUMAN & NO & 46.3 & 25.4 \\
\hline PDE2A_HUMAN & PDE7A_HUMAN & NO & 46.3 & 26 \\
\hline MK14_HUMAN & CDK8_HUMAN & NO & 46.3 & 32.2 \\
\hline BRD4_HUMAN & CDK2_HUMAN & YES & 46.1 & 31.6 \\
\hline DYR1A_HUMAN & GSK3B_HUMAN & NO & 46.1 & 29 \\
\hline CDK2_HUMAN & MYLK4_HUMAN & NO & 45.8 & 27.4 \\
\hline CBR1_HUMAN & HCK_HUMAN & NO & 45.8 & 28.8 \\
\hline PDE5A_HUMAN & PDE2A_HUMAN & NO & 45.6 & 27.5 \\
\hline CDK2_HUMAN & CHK1_HUMAN & YES & 45.5 & 28.3 \\
\hline PDE10_HUMAN & PDE4D_HUMAN & NO & 45.5 & 25.7 \\
\hline DAPK3_HUMAN & PIM1_HUMAN & NO & 45.4 & 28.9 \\
\hline CDK5_HUMAN & SRC_HUMAN & NO & 45.4 & 26.6 \\
\hline CDK2_HUMAN & ACK1_HUMAN & YES & 45.3 & 27.3 \\
\hline NR1H2_HUMAN & RORA_HUMAN & NO & 45.2 & 26.3 \\
\hline SIR1_HUMAN & SIR3_HUMAN & NO & 44.9 & 29.7 \\
\hline PDE4D_HUMAN & PDE8A_HUMAN & NO & 44.9 & 25.7 \\
\hline PAK6_HUMAN & PAK1_HUMAN & NO & 44.9 & 29.7 \\
\hline KC1G3_HUMAN & STK26_HUMAN & NO & 44.9 & 25.9 \\
\hline AVR2A_HUMAN & AAPK2_HUMAN & NO & 44.9 & 26.9 \\
\hline NEK1_HUMAN & CDK2_HUMAN & NO & 44.8 & 27.4 \\
\hline MIF_HUMAN & ESR2_HUMAN & YES & 44.7 & 25.5 \\
\hline KS6A1_HUMAN & CDK5_HUMAN & NO & 44.7 & 27.3 \\
\hline AURKA_HUMAN & ITK_HUMAN & NO & 44.7 & 26.3 \\
\hline CHK1_HUMAN & PIM1_HUMAN & YES & 44.6 & 26.6 \\
\hline RIPK2_HUMAN & FGFR4_HUMAN & NO & 44.6 & 24.6 \\
\hline
\end{tabular}




\begin{tabular}{|c|c|c|c|c|}
\hline KCC2D_HUMAN & STK26_HUMAN & NO & 44.5 & 25.1 \\
\hline CDK2_HUMAN & TTBK1_HUMAN & NO & 44.5 & 28 \\
\hline PDPK1_HUMAN & CDK2_HUMAN & YES & 44.4 & 27 \\
\hline CDKL2_HUMAN & CLK3_HUMAN & NO & 44.2 & 26.7 \\
\hline ATS5_HUMAN & MMP13_HUMAN & YES & 44.1 & 38.2 \\
\hline SRC_HUMAN & STK10_HUMAN & NO & 44.1 & 25.3 \\
\hline CDK2__HUMAN & MAPK2_HUMAN & NO & 44.1 & 26.3 \\
\hline THBG_HUMAN & TTHY_HUMAN & NO & 44 & 32 \\
\hline AVR2A_HUMAN & ULK1_HUMAN & NO & 44 & 24.9 \\
\hline ANDR_HUMAN & TTHY_HUMAN & NO & 44 & 23.1 \\
\hline JAK3_HUMAN & DAPK3_HUMAN & NO & 43.9 & 26.2 \\
\hline TNIK_HUMAN & PIM1_HUMAN & NO & 43.8 & 29 \\
\hline RXRA_HUMAN & PPARG_HUMAN & NO & 43.8 & 26.8 \\
\hline MYLK4_HUMAN & NEK1_HUMAN & NO & 43.7 & 25.2 \\
\hline HCK_HUMAN & PIM1_HUMAN & NO & 43.6 & 25.1 \\
\hline CDK2_HUMAN & NEK2_HUMAN & NO & 43.6 & 28.4 \\
\hline TNIK_HUMAN & WEE1_HUMAN & NO & 43.5 & 23.3 \\
\hline LCK_HUMAN & KSYK_HUMAN & YES & 43.3 & 29.5 \\
\hline RORG_HUMAN & CP11A_HUMAN & NO & 43.3 & 20.7 \\
\hline PDE5A_HUMAN & PDE9A_HUMAN & NO & 43.3 & 25.1 \\
\hline KCC4_HUMAN & STK26_HUMAN & NO & 43.3 & 23.7 \\
\hline STK24_HUMAN & CHK1_HUMAN & NO & 43.2 & 25.2 \\
\hline ST17B_HUMAN & VRK1_HUMAN & NO & 43.2 & 25.8 \\
\hline CLK1_HUMAN & CHK1_HUMAN & NO & 43.2 & 26.1 \\
\hline DDR1_HUMAN & KIT_HUMAN & NO & 43.1 & 29.2 \\
\hline GSK3B_HUMAN & PIM1_HUMAN & YES & 43 & 25.7 \\
\hline SRC_HUMAN & KS6A1_HUMAN & NO & 43 & 22.9 \\
\hline MCR_HUMAN & AK1D1_HUMAN & NO & 43 & 21.5 \\
\hline CDK2_HUMAN & MERTK_HUMAN & NO & 43 & 23.1 \\
\hline JAK2_HUMAN & AURKA_HUMAN & YES & 42.9 & 26 \\
\hline TTHY_HUMAN & PTGR2_HUMAN & NO & 42.9 & 24.5 \\
\hline HDAC8_HUMAN & HDAC7_HUMAN & NO & 42.9 & 26.9 \\
\hline BRD4_HUMAN & BRDT_HUMAN & NO & 42.9 & 32.7 \\
\hline PH4H_HUMAN & AL1L1_HUMAN & NO & 42.8 & 23 \\
\hline DDR1_HUMAN & MK14_HUMAN & NO & 42.6 & 24.1 \\
\hline TTHY_HUMAN & ESR1_HUMAN & NO & 42.4 & 25.9 \\
\hline KS6A1_HUMAN & KC1G3_HUMAN & NO & 42.4 & 24.6 \\
\hline CLK3_HUMAN & DYR1A_HUMAN & NO & 42.4 & 26.7 \\
\hline CDK5_HUMAN & KC1G3_HUMAN & NO & 42.4 & 27.5 \\
\hline VGFR2_HUMAN & ABL1_HUMAN & YES & 42.3 & 29 \\
\hline FABP4_HUMAN & ACS2A_HUMAN & NO & 42.3 & 18.6 \\
\hline CDK2_HUMAN & CLK1_HUMAN & NO & 42.3 & 28.6 \\
\hline RIPK2_HUMAN & D3DSX2_HUMAN & NO & 42.2 & 23.1 \\
\hline RIPK2_HUMAN & DDR1_HUMAN & NO & 42.2 & 24.6 \\
\hline DYR_HUMAN & FOLR2_HUMAN & NO & 42.1 & 31.6 \\
\hline ANDR_HUMAN & PTGIS_HUMAN & NO & 42.1 & 20.6 \\
\hline MRCKB_HUMAN & MK08_HUMAN & NO & 41.9 & 23.5 \\
\hline AVR2A_HUMAN & STK26_HUMAN & NO & 41.9 & 26.1 \\
\hline PDE3B_HUMAN & PDE4D_HUMAN & NO & 41.8 & 26.7 \\
\hline KSYK_HUMAN & BTK_HUMAN & YES & 41.7 & 25.8 \\
\hline PK3C3_HUMAN & PK3CA_HUMAN & NO & 41.7 & 24 \\
\hline CP11A_HUMAN & ADRB2_HUMAN & NO & 41.7 & 26.2 \\
\hline BRAF_HUMAN & CDK8_HUMAN & NO & 41.7 & 24.2 \\
\hline ANDR_HUMAN & THB_HUMAN & NO & 41.7 & 28.1 \\
\hline MK14_HUMAN & KC1D_HUMAN & NO & 41.6 & 24.6 \\
\hline CDKL3_HUMAN & ST17B_HUMAN & NO & 41.6 & 24.9 \\
\hline
\end{tabular}




\begin{tabular}{|c|c|c|c|c|}
\hline PDE4D_HUMAN & PDE5A_HUMAN & YES & 41.5 & 22.9 \\
\hline FABP4_HUMAN & AK1C3_HUMAN & NO & 41.3 & 37 \\
\hline CHK1_HUMAN & BMP2K_HUMAN & NO & 41.3 & 23.5 \\
\hline AURKB_HUMAN & ABL2_HUMAN & NO & 41.3 & 23.7 \\
\hline PIM1_HUMAN & CSK21_HUMAN & YES & 41.2 & 23 \\
\hline KAPCA_HUMAN & ABL1_HUMAN & NO & 41.2 & 23.4 \\
\hline PDK2_HUMAN & HS90A_HUMAN & NO & 41.1 & 21.2 \\
\hline PDE9A_HUMAN & PDE4D_HUMAN & NO & 41.1 & 24.5 \\
\hline ESR1_HUMAN & PPARG_HUMAN & NO & 41.1 & 23.8 \\
\hline BRD4_HUMAN & VGFR2_HUMAN & YES & 40.9 & 22.1 \\
\hline ATS5_HUMAN & MMP8_HUMAN & NO & 40.9 & 31.8 \\
\hline STK26_HUMAN & CDKL2_HUMAN & NO & 40.8 & 27.7 \\
\hline KS6A1_HUMAN & MERTK_HUMAN & NO & 40.7 & 22.5 \\
\hline ABL2_HUMAN & STK26_HUMAN & NO & 40.7 & 23.8 \\
\hline ABL1_HUMAN & KIT_HUMAN & YES & 40.6 & 26.3 \\
\hline ALK_HUMAN & AURKA_HUMAN & YES & 40.5 & 25.1 \\
\hline ABL1_HUMAN & NQO2_HUMAN & YES & 40.5 & 23.7 \\
\hline MERTK_HUMAN & SRC_HUMAN & NO & 40.5 & 27.1 \\
\hline BRAF_HUMAN & VGFR2_HUMAN & YES & 40.4 & 24.4 \\
\hline NR1H2_HUMAN & RORG_HUMAN & NO & 40.4 & 27.4 \\
\hline BRD4_HUMAN & PIM1_HUMAN & YES & 40.3 & 19.4 \\
\hline RET_HUMAN & CDK2_HUMAN & YES & 40.3 & 22.4 \\
\hline ULK1__HUMAN & STK26_HUMAN & NO & 40.3 & 26.3 \\
\hline PDE3B_HUMAN & PDE9A_HUMAN & NO & 40.2 & 23.7 \\
\hline MK14_HUMAN & TTBK1_HUMAN & NO & 40.2 & 23.8 \\
\hline MERTK_HUMAN & KC1G3_HUMAN & NO & 40.1 & 21.9 \\
\hline RABP2_HUMAN & RARB_HUMAN & NO & 40 & 18.5 \\
\hline PDK1_HUMAN & CP2C9_HUMAN & NO & 40 & 23.6 \\
\hline FA7_HUMAN & UROK_HUMAN & NO & 40 & 26.9 \\
\hline ABL1_HUMAN & STK10_HUMAN & NO & 40 & 22.3 \\
\hline PDE3B_HUMAN & PDE7A_HUMAN & NO & 39.9 & 23.8 \\
\hline PDPK1_HUMAN & RET_HUMAN & YES & 39.8 & 24.2 \\
\hline MK11_HUMAN & ABL1_HUMAN & NO & 39.8 & 24.9 \\
\hline CLK3_HUMAN & KCC4_HUMAN & NO & 39.8 & 24.5 \\
\hline CLK3_HUMAN & GSK3B_HUMAN & NO & 39.8 & 23.1 \\
\hline CDK2_HUMAN & HS90A_HUMAN & YES & 39.7 & 21.3 \\
\hline CAH2_HUMAN & THBG_HUMAN & NO & 39.7 & 23.8 \\
\hline NAMPT_HUMAN & NMNA3_HUMAN & NO & 39.6 & 24.5 \\
\hline MK08_HUMAN & TYK2_HUMAN & NO & 39.6 & 25 \\
\hline MIF_HUMAN & TTHY_HUMAN & NO & 39.6 & 27.1 \\
\hline ABL2_HUMAN & AURKĀHUMAN & NO & 39.6 & 22.5 \\
\hline PIM1_HUMAN & DYR1A_HUMAN & NO & 39.5 & 22 \\
\hline DDR1_HUMAN & LCK_HUMAN & NO & 39.5 & 28.3 \\
\hline AK1C2_HUMAN & FTO_HUMAN & NO & 39.5 & 26.5 \\
\hline ABL2_HUMAN & CDKL2_HUMAN & NO & 39.5 & 25.6 \\
\hline AK1C3_HUMAN & ANDR_HUMAN & YES & 39.4 & 28.8 \\
\hline KS6A1_HUMAN & KC1G1_HUMAN & NO & 39.2 & 23.3 \\
\hline TAB1_HUMAN & FAK1_HUMAN & NO & 39.1 & 23 \\
\hline KIT_HUMAN & ABL2_HUMAN & NO & 39.1 & 25.7 \\
\hline KIT_HUMAN & NQO2_HUMAN & YES & 39 & 17.7 \\
\hline SLK_HUMAN & CLK3_HUMAN & NO & 39 & 23.6 \\
\hline MK14_HUMAN & ABL1_HUMAN & YES & 38.9 & 23.1 \\
\hline THBG_HUMAN & AK1C2_HUMAN & NO & 38.9 & 23 \\
\hline SLK_HUMAN & CDKL2_HUMAN & NO & 38.9 & 23.2 \\
\hline CHK1_HUMAN & NEK2_HUMAN & NO & 38.9 & 22.7 \\
\hline SRC_HUMAN & SRPK2_HUMAN & NO & 38.8 & 22.9 \\
\hline
\end{tabular}




\begin{tabular}{|c|c|c|c|c|}
\hline CLK3_HUMAN & DYRK2_HUMAN & NO & 38.8 & 25.3 \\
\hline PPARG_HUMAN & GCR_HUMAN & YES & 38.7 & 22.8 \\
\hline FGFR4_HUMAN & KIT_HUMAN & YES & 38.6 & 26.6 \\
\hline HEXB_HUMAN & HNMT_HUMAN & NO & 38.6 & 26.3 \\
\hline NMRL1_HUMAN & TTHY_HUMAN & NO & 38.5 & 26.9 \\
\hline AVR2A_HUMAN & KCC2D_HUMAN & NO & 38.5 & 23.2 \\
\hline ABL1_HUMAN & AURKA_HUMAN & YES & 38.4 & 22.6 \\
\hline THB_HUMAN & TTHY_HUMAN & NO & 38.3 & 27.7 \\
\hline KC1G1_HUMAN & MERTK_HUMAN & NO & 38.3 & 21.2 \\
\hline ESR2_HUMAN & HS90A_HUMAN & YES & 38.2 & 18.5 \\
\hline RET_HUMAN & CBR1_HUMAN & NO & 38.2 & 22.7 \\
\hline CDKL2_HUMAN & KCC4_HUMAN & NO & 38.2 & 23.6 \\
\hline KIT_HUMAN & KSYK_HUMAN & YES & 38.1 & 25.2 \\
\hline CDK2_HUMAN & KC1G1_HUMAN & NO & 38.1 & 23.5 \\
\hline EGFR_HUMAN & AURKA_HUMAN & YES & 38 & 22 \\
\hline TNKS2_HUMAN & BRD2_HUMAN & NO & 38 & 26 \\
\hline MK10_HUMAN & TTK_HUMAN & NO & 38 & 25.7 \\
\hline MEN1_HUMAN & CP2D6_HUMAN & NO & 38 & 22.7 \\
\hline DDR1_HUMAN & FGFR4_HUMAN & NO & 38 & 26.4 \\
\hline NPC1_HUMAN & ADRB2_HUMAN & NO & 37.9 & 22 \\
\hline MRCKB_HUMAN & TYK2_HUMAN & NO & 37.9 & 25.8 \\
\hline MIF_HUMAN & MK01_HUMAN & NO & 37.9 & 21.4 \\
\hline STK2ㅎ﹎HUMAN & VRK1_HUMAN & NO & 37.8 & 21.8 \\
\hline NRK1_HUMAN & BST1_HUMAN & NO & 37.8 & 19.8 \\
\hline NAMPT_HUMAN & NRK1_HUMAN & NO & 37.8 & 25 \\
\hline KC1G3_HUMAN & CDK2_HUMAN & NO & 37.8 & 24.7 \\
\hline ERAP1_HUMAN & LKHA4_HUMAN & NO & 37.8 & 21.5 \\
\hline SRC_HUMAN & PK3CG_HUMAN & YES & 37.7 & 21.2 \\
\hline RASH_HUMAN & PPIF_HUMAN & NO & 37.6 & 22.6 \\
\hline CLK3_HUMAN & STK26_HUMAN & NO & 37.6 & 20.8 \\
\hline ALBU_HUMAN & ACS2A_HUMAN & NO & 37.6 & 29 \\
\hline MK14_HUMAN & VGFR2_HUMAN & YES & 37.5 & 23.5 \\
\hline WEE1_HUMAN & PIM1_HUMAN & NO & 37.5 & 24.3 \\
\hline PDE3B_HUMAN & PDE8A_HUMAN & NO & 37.5 & 22.2 \\
\hline MIF_HUMAN & CAH2_HUMAN & NO & 37.5 & 30.4 \\
\hline LCK_HUMAN & KIT_HUMAN & NO & 37.5 & 25.8 \\
\hline KC1G1_HUMAN & SRC_HUMAN & NO & 37.5 & 23.5 \\
\hline ACVR1_HUMAN & AAPK2_HUMAN & NO & 37.5 & 22.5 \\
\hline KC1G3_HUMAN & SRC_HUMAN & NO & 37.4 & 22.6 \\
\hline RAF1_HUMAN & BRD4_HUMAN & YES & 37.2 & 20.9 \\
\hline LCK_HUMAN & CBR1_HUMAN & NO & 37.2 & 20.3 \\
\hline FTO_HUMAN & AK1C3_HUMAN & NO & 37.2 & 23.8 \\
\hline CP11A_HUMAN & NPC1_HUMAN & NO & 37.2 & 22.9 \\
\hline AK1BA_HUMAN & NMRL1_HUMAN & NO & 37.1 & 24 \\
\hline AURKB_HUMAN & ABL1_HUMAN & YES & 37 & 22.3 \\
\hline PTGR2_HUMAN & ALBU_HUMAN & NO & 37 & 21.7 \\
\hline PIM1_HUMAN & CLK3_HUMAN & NO & 37 & 23.5 \\
\hline KC1G3_HUMAN & ABL2_HUMAN & NO & 37 & 18.5 \\
\hline AK1C3_HUMAN & PTGR2_HUMAN & NO & 37 & 21 \\
\hline ABL2_HUMAN & SLK_HUMAN & NO & 37 & 21 \\
\hline PDE5A_HUMAN & PDE4B_HUMAN & YES & 36.9 & 21 \\
\hline KIT_HUMAN & MK14_HUMAN & YES & 36.8 & 21.9 \\
\hline KIT_HUMAN & RIPK2_HUMAN & NO & 36.7 & 21.1 \\
\hline AL1L1_HUMAN & NOS2_HUMAN & NO & 36.6 & 25.8 \\
\hline CDKL3_HUMAN & VRK1_HUMAN & NO & 36.5 & 19.3 \\
\hline TNKS2_HUMAN & PAR15_HUMAN & NO & 36.4 & 21.1 \\
\hline
\end{tabular}




\begin{tabular}{|c|c|c|c|c|}
\hline DYRK2_HUMAN & PIM1_HUMAN & NO & 36.4 & 22.2 \\
\hline AK1C3_HUMAN & NMRL1_HUMAN & NO & 36.4 & 19.8 \\
\hline PK3CG_HUMAN & RET_HUMAN & YES & 36.3 & 19 \\
\hline PPARG_HUMAN & AK1C 2 _HUMAN & NO & 36.3 & 22.4 \\
\hline NEK2_HUMAN & KIT_HUMAN & NO & 36.3 & 22.5 \\
\hline CBR1_HUMAN & PK3CG_HUMAN & NO & 36.3 & 21.9 \\
\hline NEP_HUMAN & LKHA4_HUMAN & NO & 36.2 & 22 \\
\hline CLK1_HUMAN & ACK1_HUMAN & NO & 36.2 & 23.2 \\
\hline PTGR1_HUMAN & ESR1_HUMAN & NO & 36.1 & 33.3 \\
\hline ANDR_HUMAN & AK1BA_HUMAN & NO & 36.1 & 26.5 \\
\hline TTHY_HUMAN & ALBU_HUMAN & NO & 36 & 22 \\
\hline CP2C9_HUMAN & AK1C2_HUMAN & NO & 36 & 21.1 \\
\hline BRD4_HUMAN & TNKS2_HUMAN & NO & 36 & 27.9 \\
\hline AK1C3_HUMAN & PPARG_HUMAN & NO & 36 & 23 \\
\hline TTHY_HUMAN & ESR2_HUMAN & NO & 35.9 & 25.6 \\
\hline EGFR_HUMAN & TYK2_HUMAN & NO & 35.9 & 23.7 \\
\hline VGFR2_HUMAN & MET_HUMAN & YES & 35.8 & 22.6 \\
\hline CALRL_HUMAN & ST17B_HUMAN & NO & 35.8 & 20.3 \\
\hline TIE2_HUMAN & VGFR2_HUMAN & YES & 35.7 & 22.2 \\
\hline PTGR2_HUMAN & AK1C2_HUMAN & NO & 35.7 & 20.3 \\
\hline ABL2_HUMAN & KCC4_HUMAN & NO & 35.7 & 22.4 \\
\hline ACK1_HUMAN & CHK1_HUMAN & YES & 35.6 & 24.6 \\
\hline MMP12_HUMAN & MMP9_HUMAN & NO & 35.6 & 23.4 \\
\hline ATS5_HUMAN & MMP12_HUMAN & NO & 35.6 & 26 \\
\hline JAK2_HUMAN & EGFR_HUMAN & YES & 35.5 & 21.7 \\
\hline ALK_HUMAN & ABL1_HUMAN & YES & 35.5 & 24.2 \\
\hline PDE5A_HUMAN & PDE3B_HUMAN & NO & 35.5 & 19.8 \\
\hline AK1C3_HUMAN & TTHY_HUMAN & NO & 35.5 & 24.2 \\
\hline HASP_HUMAN & KAPCA_HUMAN & NO & 35.4 & 20.7 \\
\hline OXDA_HUMAN & PH4H_HUMAN & NO & 35.3 & 20.3 \\
\hline NPC1_HUMAN & NR1H2_HUMAN & NO & 35.3 & 19.8 \\
\hline AK1C2_HUMAN & ANDR_HUMAN & NO & 35.3 & 25.5 \\
\hline BRD4_HUMAN & PLK1_HUMAN & YES & 35.2 & 22.7 \\
\hline ALK_HUMAN & MET_HUMAN & YES & 35.2 & 22.2 \\
\hline TTK_HUMAN & ITK_HUMAN & NO & 35.2 & 21 \\
\hline PDE3B_HUMAN & PDE2A_HUMAN & NO & 35.2 & 22.4 \\
\hline ALBU_HUMAN & PPARG_HUMAN & NO & 35.2 & 22.1 \\
\hline ABL1_HUMAN & ERBB3_HUMAN & YES & 35.1 & 22.2 \\
\hline VRK1_HUMAN & AVR2A_HUMAN & NO & 35.1 & 22.2 \\
\hline PYGL_HUMAN & RIFK_HUMAN & NO & 35.1 & 20.8 \\
\hline NR1I2_HUMAN & NCOĀ̄1_HUMAN & NO & 35.1 & 18.4 \\
\hline KCC2D_HUMAN & VRK1_HUMAN & NO & 35.1 & 20.9 \\
\hline TNKS1_HUMAN & PAR15_HUMAN & NO & 35 & 19.6 \\
\hline CBR1_HUMAN & SRC_HUMAN & NO & 35 & 21.5 \\
\hline STK10_HUMAN & ERBB3_HUMAN & NO & 34.9 & 21.7 \\
\hline HCK_HUMAN & PK3CG_HUMAN & NO & 34.9 & 21 \\
\hline ANDR_HUMAN & THBG_HUMAN & NO & 34.9 & 23.4 \\
\hline AOFB_HUMAN & PPARG_HUMAN & YES & 34.8 & 21.6 \\
\hline KPCA_HUMAN & JAK3_HUMAN & NO & 34.8 & 22.7 \\
\hline ITK_HUMAN & VGFR2_HUMAN & NO & 34.8 & 22.9 \\
\hline DCK_HUMAN & ATAD2_HUMAN & NO & 34.8 & 22.3 \\
\hline CBR1_HUMAN & BMX_HUMAN & NO & 34.8 & 21.7 \\
\hline AK1C3_HUMAN & CP2C9_HUMAN & NO & 34.8 & 19.1 \\
\hline PK3CA_HUMAN & MTOR_HUMAN & YES & 34.7 & 19.7 \\
\hline ADRB2_HUMAN & NR1H2_HUMAN & NO & 34.7 & 30.6 \\
\hline PDPK1_HUMAN & F16P1_HUMAN & NO & 34.6 & 19.5 \\
\hline
\end{tabular}




\begin{tabular}{|c|c|c|c|c|}
\hline PDE8A_HUMAN & PDE5A_HUMAN & NO & 34.6 & 19.5 \\
\hline PDE8A_HUMAN & PDE2A_HUMAN & NO & 34.2 & 20.4 \\
\hline PDE4D_HUMAN & PDE2A_HUMAN & NO & 34.2 & 20.8 \\
\hline NOS1_HUMAN & AL1L1_HUMAN & NO & 34.2 & 20.3 \\
\hline NMNA3_HUMAN & CD38_HUMAN & NO & 34.2 & 20.5 \\
\hline KC1G3_HUMAN & CDKL2_HUMAN & NO & 34.2 & 22.2 \\
\hline KC1G3_HUMAN & SLK_HUMAN & NO & 34.2 & 20 \\
\hline VTDB_HUMAN & VDR_HUMAN & NO & 33.9 & 20.6 \\
\hline TNKS2_HUMAN & AURKA_HUMAN & NO & 33.9 & 21.7 \\
\hline PH4H_HUMAN & NOS3_HUMAN & NO & 33.9 & 21.9 \\
\hline EGLN1_HUMAN & FTO_HUMAN & NO & 33.9 & 21.9 \\
\hline PH4H_HUMAN & NOS1_HUMAN & NO & 33.8 & 16.9 \\
\hline CDK9_HUMAN & BRD4_HUMAN & NO & 33.8 & 17.5 \\
\hline NMRL1_HUMAN & ANDR_HUMAN & NO & 33.7 & 19.9 \\
\hline PH4H_HUMAN & NOS2_HUMAN & NO & 33.6 & 17.3 \\
\hline JAK1_HUMAN & PKN1_HUMAN & NO & 33.6 & 19.7 \\
\hline ERR3_HUMAN & EST1_HUMAN & NO & 33.6 & 18.7 \\
\hline CP3A4_HUMAN & BACE1_HUMAN & NO & 33.6 & 22.3 \\
\hline CHK1_HUMAN & EGFR_HUMAN & YES & 33.3 & 20.2 \\
\hline MTOR_HUMAN & PK3C3_HUMAN & NO & 33.3 & 19.5 \\
\hline MERTK_HUMAN & SRPK2_HUMAN & NO & 33.3 & 19.3 \\
\hline AK1C3_HUMAN & ALBU_HUMAN & NO & 33.3 & 21.4 \\
\hline BRD4_HUMAN & BRD9_HUMAN & NO & 33.2 & 21.1 \\
\hline ACS2A_HUMAN & AK1C3_HUMAN & NO & 33.2 & 19 \\
\hline TYK2_HUMAN & PKN1_HUMAN & NO & 33.1 & 21.3 \\
\hline FTO_HUMAN & THBG_HUMAN & NO & 33.1 & 20.7 \\
\hline PARP2_HUMAN & TNKS2_HUMAN & NO & 33 & 22.2 \\
\hline OXDA_HUMAN & SYFM_HUMAN & NO & 32.9 & 21.7 \\
\hline NOS2_HUMAN & TPH1_HUMAN & NO & 32.9 & 19.3 \\
\hline PKN1_HUMAN & JAK2_HUMAN & NO & 32.8 & 19.8 \\
\hline MMP16_HUMAN & ATS5_HUMAN & NO & 32.8 & 21.2 \\
\hline ROS1_HUMAN & MET_HUMAN & YES & 32.7 & 20.8 \\
\hline SRPK2_HUMAN & KS6A1_HUMAN & NO & 32.7 & 20.2 \\
\hline KC1G3_HUMAN & SRPK2_HUMAN & NO & 32.7 & 18.2 \\
\hline FTO_HUMAN & AK1BA_HUMAN & NO & 32.5 & 22 \\
\hline NQO2_HUMAN & DDR1_HUMAN & NO & 32.4 & 22.5 \\
\hline KC1G3_HUMAN & CLK3_HUMAN & NO & 32.4 & 20.5 \\
\hline PPARG_HUMAN & PTGR2_HUMAN & NO & 32.3 & 19.4 \\
\hline RORG_HUMAN & NPC1_HUMAN & NO & 32.2 & 20.9 \\
\hline JAK3_HUMAN & PKN1_HUMAN & NO & 32.1 & 21.8 \\
\hline BRD4_HUMAN & JAK2_HUMAN & YES & 31.8 & 18.7 \\
\hline VGFR2_HUMAN & CDK8_HUMAN & NO & 31.8 & 20.3 \\
\hline CLK3_HUMAN & ABL2_HUMAN & NO & 31.8 & 20.5 \\
\hline ADRB2_HUMAN & RORA_HUMAN & NO & 31.8 & 18.8 \\
\hline CP46A_HUMAN & RORA_HUMAN & NO & 31.7 & 17.9 \\
\hline ANDR_HUMAN & RET_HUMAN & YES & 31.6 & 17.1 \\
\hline ANDR_HUMAN & HCK_HUMAN & NO & 31.4 & 19.8 \\
\hline AK1C3_HUMAN & THBG_HUMAN & NO & 31.4 & 20 \\
\hline NOS3_HUMAN & AL1L1_HUMAN & NO & 31.3 & 19.6 \\
\hline CP11A_HUMAN & RORA_HUMAN & NO & 31.2 & 18.7 \\
\hline AK1C2_HUMAN & ALBU_HUMAN & NO & 31.2 & 18.3 \\
\hline AMYP_HUMAN & TTHY_HUMAN & NO & 31 & 22.1 \\
\hline TTHY_HUMAN & ALDR_HUMAN & NO & 30.9 & 25.8 \\
\hline PPARG_HUMAN & TTHY_HUMAN & NO & 30.9 & 19.1 \\
\hline NAMPT_HUMAN & BST1_HUMAN & NO & 30.9 & 20.4 \\
\hline PARP1_HUMAN & TNKS2_HUMAN & NO & 30.7 & 18.9 \\
\hline
\end{tabular}




\begin{tabular}{ccccc} 
BRAF_HUMAN & BRD4_HUMAN & YES & 30.6 & 17.1 \\
FTO_HUMAN & ANDR_HUMAN & NO & 30.6 & 20.1 \\
NAMPT_HUMAN & CD38_HUMAN & NO & 30.4 & 19.1 \\
AK1BA_HUMAN & THBG_HUMAN & NO & 30.2 & 20.4 \\
NR1H2_HUMAN & CP11A_HUMAN & NO & 29.9 & 16.7 \\
ANDR_HUMAN & ALBU_HUMAN & NO & 29.9 & 21.7 \\
TTHY_HUMAN & FTO_HUMAN & NO & 29.7 & 18.9 \\
SYFM_HUMAN & PH4H_HUMAN & NO & 29.2 & 19.1 \\
NQO2_HUMAN & MK14_HUMAN & YES & 29 & 19.7 \\
NPC1_HUMAN & RORA_HUMAN & NO & 29 & 17 \\
ACE_HUMAN & LKHA4_HUMAN & NO & 28.7 & 16.5 \\
BRD4_HUMAN & CBP_HUMAN & NO & 27.8 & 19.4 \\
RXRA_HUMAN & FTO_HUMAN & NO & 26.5 & 19.1 \\
\hline
\end{tabular}




\section{REFERENCES}

(1) Norman, B. H.; Dodge, J. A.; Richardson, T. I.; Borromeo, P. S.; Lugar, C. W.; Jones, S. A.; Chen, K.; Wang, Y.; Durst, G. L.; Barr, R. J.; et al. Benzopyrans Are Selective Estrogen Receptor $\beta$ Agonists with Novel Activity in Models of Benign Prostatic Hyperplasia. J. Med. Chem. 2006, 49 (21), 6155-6157.

(2) Mewshaw, R. E.; Edsall Richard J.; Yang, C.; Manas, E. S.; Xu, Z. B.; Henderson, R. A.; Keith James C.; Harris, H. A. ER $\beta$ Ligands. 3. Exploiting Two Binding Orientations of the 2-Phenylnaphthalene Scaffold To Achieve ER $\beta$ Selectivity. J. Med. Chem. 2005, 48 (12), 3953-3979. 